\title{
Robust Discretisation and a Posteriori Control for Strongly Oscillating Solutions of the Stationary Schrödinger Equation
}

\author{
Willy Dörfler
}

\begin{abstract}
We consider an example of a boundary value problem on an interval where the solution can show strong oscillations. In order to solve such a problem numerically, standard methods require meshes that resolve these oscillations and will thus need a prohibitively large number of unknowns. In our approach we use special problem dependent basis functions in the finite element method and provide an analysis for a priori and a posteriori bounds. In this way we can construct an efficient approximation method for the solution of such boundary value problems.
\end{abstract}

2010 Mathematical subject classification: 65N12; 65N15; 65N30; 65N80.

Keywords: A posteriori error estimates; a priori error estimates; boundary value problems; computable error bounds; exponential fitting; Schrödinger equation.

\section{Introduction}

The numerical solution of linear elliptic boundary value problems that define a symmetric positive form is very elaborated in terms of a priori and a posteriori analysis. Also for the singularly perturbed operators (when the operators' coefficients become very small or large) one can describe robust methods. However, if one studies these problems with a negative potential, especially when it is large in modulus, standard methods face some difficulties. For example, the solutions of such problems may exhibit strong oscillations and an approximation by piecewise polynomials will require small computational cells that resolve these oscillations. However, this will result in a very expensive method. In this work we will, for the one-dimensional case, perform the Galerkin discretisation with local Green's functions that have been used and analysed up to now mainly in case of positive coefficients [7] [19] [1] [5]. For negative coefficients the basis functions are strongly oscillating and the question is to which extend they can approximate the solution of the problem. Since the setting is that of a general finite element method, we can follow the general guideline to prove a priori and a posteriori error estimates once we provided the necessary interpolation estimates. This work generalises the corresponding attempt in [3, Ch. 4.1] to non-constant coefficients and a posteriori error estimations and mesh-refinement.

Note, that the idea of exponential fitting of such type of equations have been considered in the context of finite difference methods, see for example [8] and the literature cited therein. However, our methods and results are quite different from this approach.

Willy Dörfler

Institut für Angewandte und Numerische Mathematik 2, Karlsruher Institut für Technologie (KIT), 76128 Karlsruhe, Germany E-mail: willy.doerfler@kit.edu 
Oscillating basis functions have also been used in a similar type of problem, the Helmholtz equation with constant coefficients and with wave-number dependent Robin boundary conditions. The methods and results are different to those used here [11] [10] [13] [20].

The techniques are essentially for the one-dimensional case. We think that the method might be of interest for the time evolution of 1D oscillating pulses. Furthermore, it has been demonstrated in [5] how to use such one-dimensional results on tensorial grids. In [3, Ch. 7.2] [15] it is demonstrated that a local choice of a number of $1 \mathrm{D}$ plane waves can be used to approximate multi-dimensional problems.

\section{The stationary Schrödinger equation}

The stationary Schrödinger equation is the boundary value problem

$$
\begin{aligned}
L(\gamma) u:=-u^{\prime \prime}+\gamma u=f & & \text { in } \Omega:=(0,1), \\
u(0)=u(1)=0 & & \text { in } \partial \Omega=\{0,1\}
\end{aligned}
$$

with a bounded function $\gamma: \Omega \rightarrow \mathbb{R}$. Note that any bounded interval can be transformed (affine linearly) to $(0,1)$ and nonhomogeneous boundary values can be removed by substraction of a suitable linear function from $u$.

The weak form of this boundary value problem results from multiplying (2.1) by $v \in$ $C_{0}^{\infty}(\Omega)$ and integration by parts

$$
B(u, v):=\int_{\Omega}\left\{u^{\prime} v^{\prime}+\gamma u v\right\}=F(v):=\int_{\Omega} f v \quad \text { for all } v \in C_{0}^{\infty}(\Omega) .
$$

To formulate this problem on a Hilbert space we let

$$
\kappa:=\sqrt{|\gamma|}, \quad \kappa_{*}:=\max \{\kappa, \pi\}, \quad \gamma_{*}:=\kappa_{*}^{2},
$$

and define for $G \subseteq \Omega$

$$
\|v\|_{\kappa ; G}:=\left(\left\|v^{\prime}\right\|_{L^{2}(G)}^{2}+\left\|\kappa_{*} v\right\|_{L^{2}(G)}^{2}\right)^{1 / 2} .
$$

Assuming that $\gamma$ is a bounded function, we take $V:=H_{0}^{1}(\Omega)$ equipped with the norm $\|\cdot\|_{\kappa ; \Omega}$ and observe immediately the continuity of the bilinear form $B$ on $V \times V$

$$
|B(v, w)| \leqslant \int_{\Omega}\left\{\left|v^{\prime}\right|\left|w^{\prime}\right|+|\gamma||v||w|\right\} \leqslant\|v\|_{\kappa ; \Omega}\|w\|_{\kappa ; \Omega} .
$$

In case $\gamma$ is strictly positive we have furthermore for all $v \in V$

$$
\begin{aligned}
|B(v, v)| & =\int_{\Omega}\left\{\left|v^{\prime}\right|^{2}+\gamma|v|^{2}\right\} \geqslant \int_{\Omega}\left|v^{\prime}\right|^{2}+\int_{\Omega \cap\{\kappa \geqslant \pi\}} \gamma|v|^{2} \\
& \geqslant \frac{1}{2} \int_{\Omega}\left|v^{\prime}\right|^{2}+\frac{1}{2} \pi^{2} \int_{\Omega \cap\{\kappa<\pi\}}|v|^{2}+\int_{\Omega \cap\{\kappa \geqslant \pi\}} \gamma|v|^{2} \geqslant \frac{1}{2}\|v\|_{\kappa ; \Omega}^{2},
\end{aligned}
$$

where we have used Poincaré's inequality $\|v\|_{L^{2}(\Omega)} \leqslant(1 / \pi)\left\|v^{\prime}\right\|_{L^{2}(\Omega)}$ and $\{\kappa<\pi\}$ as an abbreviation for the set $\{x \in \Omega: \kappa(x)<\pi\}$, etc. This inequality, called coercivity, guarantees unique solvability of problem (2.3) for any continuous $F: V \rightarrow \mathbb{R}$ on $V$ [6, Lem. 2.2]. This, 
however, cannot be applied for $\gamma$ with (not necessarily small) negative values and it is in fact not true in general. Thus we will have to assume invertibility of $L(\gamma)$ for the given data $\gamma$, stated as the inf-sup-condition

$$
\inf _{v \in V \backslash\{0\}} \sup _{w \in V \backslash\{0\}}\left\{\frac{B(v, w)}{\|v\|_{\kappa ; \Omega}\|w\|_{\kappa ; \Omega}}\right\} \geqslant c_{\gamma}
$$

for some positive constant $c_{\gamma}>0[6$, Thm. 2.6]. We finally observe that $F$ satisfies the bound

$$
|F(v)| \leqslant \int_{\Omega}|f||v|=\int_{\Omega} \frac{1}{\kappa_{*}}|f| \kappa_{*}|v| \leqslant\left\|\frac{1}{\kappa_{*}} f\right\|_{L^{2}(\Omega)}\|v\|_{\kappa ; \Omega} .
$$

In summary, we have the following existence and stability result.

Theorem 2.1. Assume that for given $\gamma \in L^{\infty}(\Omega)$ assumption (2.4) holds true. Then there exists a unique solution $u \in V$ of (2.3) for any $f \in L^{2}(\Omega)$ and it satisfies the bound

$$
\|u\|_{\kappa ; \Omega} \leqslant \frac{1}{c_{\gamma}}\left\|\frac{1}{\kappa_{*}} f\right\|_{L^{2}(\Omega)} .
$$

In case $\gamma$ is strictly positive assumption (2.4) is satisfied with $c_{\gamma}=1 / 2$.

\section{The finite element method}

\subsection{Galerkin method}

For a numerical approximation we choose the Galerkin method that consists of choosing a finite dimensional space $V_{h} \subset V$ and to formulate the discrete boundary value problem as

$$
B_{h}\left(u_{h}, v_{h}\right):=\int_{\Omega}\left\{u_{h}^{\prime} v_{h}^{\prime}+\gamma_{h} u_{h} v_{h}\right\}=F_{h}\left(v_{h}\right):=\int_{\Omega} f_{h} v_{h} \quad \text { for all } v_{h} \in V_{h},
$$

where $\gamma_{h}$ and $f_{h}$ are suitable approximations to $\gamma$ and $f$, respectively. $B$ and $B_{h}$ are connected via

$$
B_{h}\left(v_{h}, w_{h}\right)=B\left(v_{h}, w_{h}\right)+\int_{\Omega}\left(\gamma_{h}-\gamma\right) v_{h} w_{h}
$$

Since for all $v_{h}, w_{h} \in V_{h}$

$$
\left|\int_{\Omega}\left(\gamma-\gamma_{h}\right) v_{h} w_{h}\right| \leqslant \int_{\Omega} \frac{\left|\gamma-\gamma_{h}\right|}{\gamma_{*}} \kappa_{*}\left|v_{h}\right| \kappa_{*}\left|w_{h}\right| \leqslant \Gamma\left(\gamma, \gamma_{h}\right)\left\|v_{h}\right\|_{\kappa ; \Omega}\left\|w_{h}\right\|_{\kappa ; \Omega},
$$

with $\Gamma\left(\gamma, \gamma_{h}\right):=\left\|\left(\gamma-\gamma_{h}\right) / \gamma_{*}\right\|_{L^{\infty}(\Omega)}$, we find that

$$
\left|B_{h}\left(v_{h}, w_{h}\right)\right| \leqslant\left(1+\Gamma\left(\gamma, \gamma_{h}\right)\right)\left\|v_{h}\right\|_{\kappa ; \Omega}\left\|w_{h}\right\|_{\kappa ; \Omega} .
$$

However, a discrete inf-sup-condition

$$
\inf _{v_{h} \in V_{h} \backslash\{0\}} \sup _{w_{h} \in V_{h} \backslash\{0\}}\left\{\frac{B_{h}\left(v_{h}, w_{h}\right)}{\left\|v_{h}\right\|_{\kappa ; \Omega}\left\|w_{h}\right\|_{\kappa ; \Omega}}\right\} \geqslant \beta c_{\gamma}
$$


for some constant $\beta \in(0,1)$ that is independent of the dimension of $V_{h}$ cannot be deduced from (2.4) for general coefficients $\gamma$ and spaces $V_{h}$ (although it will be true asymptotically for $h \rightarrow 0$ under reasonable conditions [16]). In the following we will assume that the condition (3.2) is satisfied. In this situation the application of the Strang lemma yields the a priori error bound [6, Lem. 2.27]

$$
\begin{aligned}
\left\|u-u_{h}\right\|_{\kappa ; \Omega} \leqslant( & \left.+\frac{1+\Gamma\left(\gamma, \gamma_{h}\right)}{\beta c_{\gamma}}\right) \inf _{v_{h} \in V_{h}}\left\{\left\|u-v_{h}\right\|_{\kappa ; \Omega}\right\} \\
& +\frac{1}{\beta c_{\gamma}}\left\|\frac{\gamma-\gamma_{h}}{\gamma_{*}} \kappa_{*} u\right\|_{L^{2}(\Omega)}+\frac{1}{\beta c_{\gamma}}\left\|\frac{1}{\kappa_{*}}\left(f-f_{h}\right)\right\|_{L^{2}(\Omega)} .
\end{aligned}
$$

\subsection{Standard finite element spaces}

Let $\mathcal{G}=\left\{x_{i}: i=0, \ldots, N+1\right\}$ be a set of points with $0=x_{0}<x_{1}<\cdots<x_{N}<x_{N+1}=1$ and $\mathcal{K}=\left\{K_{i}:=\left[x_{i-1}, x_{i}\right]: i=0, \ldots, N+1\right\}$ be a decomposition of $\bar{\Omega}$ into intervals. We define $h_{i}:=\left|K_{i}\right|$ and $h$ to be the piecewise constant function $h(x):=h_{i}$ for $x \in K_{i}$. In a standard finite element discretisation the space $V_{h}$ would consist of continuous piecewise (with respect to the decomposition) polynomials of a, say constant in $\Omega$, degree $p$. For a stable discretisation, when (3.2) holds, the error estimate follows from (3.3) with the interpolation estimate

$$
\inf _{v_{h} \in V_{h}}\left\{\left\|u-v_{h}\right\|_{\kappa ; \Omega}\right\} \leqslant \min _{l=1, \ldots, p} C_{l}\left(\frac{\kappa h}{l}\right)^{l}
$$

sufficient regularity assumed [17, Thm. 3.17] [14, p. 24]. Moreover, this bound requires $\kappa h<l$ for some $l \in\{1, \ldots, p\}$. This is a very strong requirement on the resolution of the grid for large $\kappa$ and a problem in many applications. Our aim is to create a finite element space with approximation properties that are better suited to this problem.

\subsection{Exponentially fitted finite element spaces}

Let $\bar{\gamma}_{h}$ be a piecewise constant function on the decomposition $\mathcal{K}$. By $\bar{\gamma}_{h, i}$ we denote the restriction of $\bar{\gamma}_{h}$ on $K_{i}$. We define

$$
V_{h}^{1}:=\operatorname{span}\left\{\psi_{i}: i=1, \ldots, N\right\}
$$

where the basis functions $\psi_{i}$ are uniquely determined by the local boundary value problems

$$
\begin{aligned}
L\left(\bar{\gamma}_{h}\right) \psi_{i}=0 & \text { on } \cup_{j=1}^{N+1} K_{j}, \\
\psi_{i}\left(x_{j}\right)=\delta_{i j} & \text { for } j=0, \ldots, N+1 .
\end{aligned}
$$

It is immediately seen that $\psi_{i}$ is zero outside the interval $K_{i} \cup K_{i+1}$ for $i=1, \ldots, N$. On the interval $K_{i}$ we have explicitly

$$
\psi_{i-1}(x)=\left\{\begin{array}{ll}
\frac{\sin \left(\bar{\kappa}_{h, i}\left(x_{i}-x\right)\right)}{\sin \left(\bar{\kappa}_{h, i} h_{i}\right)} & \text { for } \bar{\gamma}_{h, i}<0, \\
\frac{x_{i}-x}{h_{i}} & \text { for } \bar{\gamma}_{h, i}=0, \\
\frac{\sinh \left(\bar{\kappa}_{h, i}\left(x_{i}-x\right)\right)}{\sinh \left(\bar{\kappa}_{h, i} h_{i}\right)} & \text { for } \bar{\gamma}_{h, i}>0,
\end{array} \quad \psi_{i}(x)= \begin{cases}\frac{\sin \left(\bar{\kappa}_{h, i}\left(x-x_{i-1}\right)\right)}{\sin \left(\bar{\kappa}_{h, i} h_{i}\right)} & \text { for } \bar{\gamma}_{h, i}<0, \\
\frac{x-x_{i-1}}{h_{i}} & \text { for } \bar{\gamma}_{h, i}=0, \\
\frac{\sinh \left(\bar{\kappa}_{h, i}\left(x-x_{i-1}\right)\right)}{\sinh \left(\bar{\kappa}_{h, i} h_{i}\right)} & \text { for } \bar{\gamma}_{h, i}>0,\end{cases}\right.
$$


where $\bar{\kappa}_{h, i}:=\sqrt{\left|\bar{\gamma}_{h, i}\right|}$ and where $\bar{\kappa}_{h, i} h_{i} \notin \pi \mathbb{N}$ has to be assumed in case of $\bar{\gamma}_{h, i}<0$. We further define the enhanced space

$$
V_{h}^{2}:=V_{h}^{1} \cup \operatorname{span}\left\{\psi_{i-\frac{1}{2}}: i=1, \ldots, N+1\right\},
$$

with $\psi_{i-1 / 2}$ given as the unique solution of

$$
\begin{aligned}
L\left(\bar{\gamma}_{h}\right) \psi_{i-\frac{1}{2}} & =\delta_{i j} & & \text { on } K_{j} \text { for } j=1, \ldots, N+1, \\
\psi_{i-\frac{1}{2}}\left(x_{j}\right) & =0 & & \text { for } j=0, \ldots, N+1 .
\end{aligned}
$$

Clearly, $\psi_{i-1 / 2}$ is compactly supported inside $K_{i}$ and it can easily be computed using the functions $\left\{\psi_{i-1}, \psi_{i}\right\}$ on $K_{i}$ defined above. In fact, for $x \in K_{i}$,

$$
\psi_{i-\frac{1}{2}}(x)= \begin{cases}\frac{1}{\bar{\gamma}_{h, i}}\left(1-\psi_{i-1}(x)-\psi_{i}(x)\right) & \text { for } \bar{\gamma}_{h, i} \neq 0 \\ \frac{1}{2}\left(x-x_{i-1}\right)\left(x_{i}-x\right) & \text { for } \bar{\gamma}_{h, i}=0\end{cases}
$$

This type of basis functions are also called local Green's functions [7].

\subsection{The discrete problem}

In general $u_{h}$ will be of the form

$$
u_{h}(x)=\sum_{i=1}^{N} a_{i} \psi_{i}(x)+\sum_{j=1}^{N+1} b_{j} \psi_{j-\frac{1}{2}}(x)
$$

for coefficients $a_{i}, b_{j}$ to be determined. For $u_{h} \in V_{h}^{1}$ we have $b_{j}=0$ for all $j$. To solve the problem for $u_{h} \in V_{h}^{1}$ we end up with a linear system with a tridiagonal matrix $A=$ $\left[A_{i j}\right]_{i, j=1, \ldots, N}$ given by

$$
A_{i j}=\int_{\Omega}\left\{\psi_{j}^{\prime} \psi_{i}^{\prime}+\gamma_{h} \psi_{j} \psi_{i}\right\}=-\left[\psi_{j}^{\prime} \psi_{i}\right]_{x_{i}}+\int_{\Omega} \psi_{i} L\left(\gamma_{h}\right) \psi_{j}=-\left[\psi_{j}^{\prime}\right]_{x_{i}}+\int_{\Omega}\left(\gamma_{h}-\bar{\gamma}_{h}\right) \psi_{j} \psi_{i}
$$

since $L\left(\bar{\gamma}_{h}\right) \psi_{j}=0$ on each interval. Here, $[g]_{x_{i}}$ denotes the jump of a piecewise continuous function $g$ in $x_{i}:[g]_{x_{i}}:=\lim _{s \rightarrow 0+}\left(g\left(x_{i}+s\right)-g\left(x_{i}-s\right)\right)$.

For the problem in $V_{h}^{2}$ we find in case $j=1, \ldots, N$

$$
\begin{aligned}
A_{i-1 / 2, j} & =\int_{\Omega}\left\{\psi_{j}^{\prime} \psi_{i-1 / 2}^{\prime}+\gamma_{h} \psi_{j} \psi_{i-1 / 2}\right\}=-\left[\psi_{j}^{\prime} \psi_{i-1 / 2}\right]_{x_{i}}+\int_{\Omega} \psi_{i-1 / 2} L\left(\gamma_{h}\right) \psi_{j} \\
& =\int_{\Omega}\left(\gamma_{h}-\bar{\gamma}_{h}\right) \psi_{j} \psi_{i-1 / 2}
\end{aligned}
$$

and likewise

$$
\begin{aligned}
A_{i-1 / 2, i-1 / 2} & =\int_{\Omega}\left\{\psi_{i-1 / 2}^{\prime} \psi_{i-1 / 2}^{\prime}+\gamma_{h} \psi_{i-1 / 2} \psi_{i-1 / 2}\right\}=\int_{\Omega} \psi_{i-1 / 2} L\left(\gamma_{h}\right) \psi_{i-1 / 2} \\
& =\int_{\Omega} \psi_{i-1 / 2}+\int_{\Omega}\left(\gamma_{h}-\bar{\gamma}_{h}\right) \psi_{i-1 / 2} \psi_{i-1 / 2}
\end{aligned}
$$

since $L\left(\bar{\gamma}_{h}\right) \psi_{i-1 / 2}=1$ on $K_{i}$. The right-hand side of the system is a vector $F$ with components

$$
F_{i}=\int_{\Omega} f_{h} \psi_{i}, \quad F_{i-1 / 2}=\int_{\Omega} f_{h} \psi_{i-1 / 2} .
$$


In the special case $\gamma_{h}=\bar{\gamma}_{h}$ and $f_{h}=\bar{f}_{h}$ we obtain $A_{i j}=-\left[\psi_{j}^{\prime}\right]_{x_{i}}, A_{i-1 / 2, j}=0$ (the equations for $a_{i}, b_{i}$ decouple), while $A_{i-1 / 2, i-1 / 2}=\int_{K_{i}} \psi_{i-1 / 2}$ and $\int_{\Omega} f_{h} \psi_{i-1 / 2}=\bar{f}_{h, i} \int_{\Omega} \psi_{i-1 / 2}$ yield $b_{i}=\bar{f}_{h, i}$ directly.

Definition 3.1. For a given piecewise constant function $\bar{\gamma}_{h}$ on a decomposition $\mathcal{K}$ we define the index set

$$
\bar{\Lambda}_{-}:=\left\{i \in\{1, \ldots, N+1\}: \bar{\gamma}_{h, i}<0\right\}
$$

and the interpolation constant

$$
C_{\mathrm{I}}:= \begin{cases}\max \left\{\frac{1}{\left|\sin \left(\bar{\kappa}_{h, i} h_{i}\right)\right|}: i \in \bar{\Lambda}_{-}, \bar{\kappa}_{h, i} h_{i} \geqslant \pi / 2\right\} & \text { if the set is not empty } \\ 1 & \text { otherwise. }\end{cases}
$$

We end this section with an a priori error estimate for this kind of discretisation (in contrast to the estimate given in Section 3.2).

Theorem 3.1. Let $u \in H^{2}(\Omega)$ be a solution to $(2.1)-(2.2)$ and $u_{h}^{(1)} \in V_{h}^{1}$ and $u_{h}^{(2)} \in V_{h}^{2}$ be the respective solutions of the discrete problem (3.1) on a grid where $C_{\mathrm{I}}$ given by (3.7) is bounded. Then,

$$
\begin{gathered}
\left\|u-u_{h}^{(\ell)}\right\|_{\kappa ; \Omega} \leqslant 4 \\
C_{\mathrm{I}}\left(1+\frac{1+\Gamma\left(\gamma, \gamma_{h}\right)}{\beta c_{\gamma}}\right)\left(1+\Gamma\left(\bar{\kappa}_{h}, \kappa\right)\right)\left(\|h f\|_{L^{2}(\Omega)}+\left\|h\left(\gamma-\bar{\gamma}_{h}\right) u\right\|_{L^{2}(\Omega)}\right) \\
+\frac{1}{\beta c_{\gamma}}\left\|\frac{\gamma-\gamma_{h}}{\gamma_{*}} \kappa_{*} u\right\|_{L^{2}(\Omega)}+\frac{1}{\beta c_{\gamma}}\left\|\frac{1}{\kappa_{*}}\left(f-f_{h}\right)\right\|_{L^{2}(\Omega)}
\end{gathered}
$$

for $\ell \in\{1,2\}$, and especially for $\ell=2$ also

$$
\begin{gathered}
\left\|u-u_{h}^{(2)}\right\|_{\kappa ; \Omega} \leqslant 4 \\
C_{\mathrm{I}}\left(1+\frac{1+\Gamma\left(\gamma, \gamma_{h}\right)}{\beta c_{\gamma}}\right)\left(1+\Gamma\left(\bar{\kappa}_{h}, \kappa\right)\right)\left(\left\|h^{2} f^{\prime}\right\|_{L^{2}(\Omega)}+\left\|h^{2}\left(\left(\gamma-\bar{\gamma}_{h}\right) u\right)_{h}^{\prime}\right\|_{L^{2}(\Omega)}\right) \\
+\frac{1}{\beta c_{\gamma}}\left\|\frac{\gamma-\gamma_{h}}{\gamma_{*}} \kappa_{*} u\right\|_{L^{2}(\Omega)}+\frac{1}{\beta c_{\gamma}}\left\|\frac{1}{\kappa_{*}}\left(f-f_{h}\right)\right\|_{L^{2}(\Omega)} .
\end{gathered}
$$

$(.)_{h}^{\prime}$ denotes the piecewise derivative with respect to $\mathcal{K}$.

Proof. Note first, that a grid with the required property can be obtained by the construction presented in Section 5.4. Using the error estimate (3.3), the remaining task is to estimate the infimum after insertion of a suitable interpolant. Let us consider the case of a discrete solution in $V_{h}^{1}$. Then, by Theorem 4.1 and a further triangle inequality,

$$
\left\|u-I_{h}^{1} u\right\|_{\bar{\kappa}_{h} ; \Omega} \leqslant 4 C_{\mathrm{I}}\left\|h L\left(\bar{\gamma}_{h}\right) u\right\|_{L^{2}(\Omega)} \leqslant 4 C_{\mathrm{I}}\left(\|h f\|_{L^{2}(\Omega)}+\left\|h\left(\gamma-\bar{\gamma}_{h}\right) u\right\|_{L^{2}(\Omega)}\right)
$$

and this shows the first estimate for $\ell=1$ with $\left\|u-I_{h}^{1} u\right\|_{\kappa ; \Omega} \leqslant\left(1+\Gamma\left(\bar{\kappa}_{h}, \kappa\right)\right)\left\|u-I_{h}^{1} u\right\|_{\bar{\kappa}_{h} ; \Omega}$. The assertion for $\ell=2$ works likewise with Theorem 4.3.

\section{Interpolation estimates}

We study suitable linear continuous operators $I_{h}^{k}: V \rightarrow V_{h}^{k}$ (for $k \in\{1,2\}$ ) and give estimates for $\left\|v-I_{h}^{k} v\right\|_{L^{2}(\Omega)}$ in terms of $v$ in $H^{1}(\Omega)$ or $H^{2}(\Omega)$. For $v \in H^{1}(\Omega)$ let us define

$$
I_{h}^{1} v(x):=v\left(x_{i-1}\right) \psi_{i-1}(x)+v\left(x_{i}\right) \psi_{i}(x) \quad \text { for } x \in K_{i}, i \in\{1, \ldots, N+1\}
$$


with the basis functions from (3.4). Note that for constant $v=v_{0}$ on $K_{i}$ we get from (3.5) $I_{h}^{1} v(x)=v_{0}\left(\psi_{i-1}(x)+\psi_{i}(x)\right)=v_{0}\left(1-\bar{\gamma}_{h, i} \psi_{i-1 / 2}(x)\right)$ on $K_{i}$ which not equals $v_{0}$. On the enhanced space $V_{h}^{2}$ we can define for $v \in H^{1}(\Omega)$

$$
I_{h}^{2} v(x):=I_{h}^{1} v(x)+\frac{\bar{\gamma}_{h, i}}{2}\left(v\left(x_{i-1}\right)+v\left(x_{i}\right)\right) \psi_{i-1 / 2}(x) \quad \text { for } x \in K_{i}, i \in\{1, \ldots, N+1\} .
$$

For a constant function $v=v_{0}$ on $K_{i}$ we get $I_{h}^{2} v(x)=v_{0}\left(1-\bar{\gamma}_{h, i} \psi_{i-1 / 2}(x)\right)+v_{0} \bar{\gamma}_{h, i} \psi_{i-1 / 2}(x)=$ $v_{0}$ on $K_{i}$. Alternatively, we define for $v \in H^{2}(\Omega)$

$$
\widetilde{I}_{h}^{2} v(x):=I_{h}^{1} v(x)+\left(\frac{1}{h_{i}} \int_{K_{i}} L\left(\bar{\gamma}_{h, i}\right) v\right) \psi_{i-1 / 2}(x) \quad \text { for } x \in K_{i}, i \in\{1, \ldots, N+1\},
$$

that also has the property $\widetilde{I}_{h}^{2} v_{0}=v_{0}$ for constant $v_{0}$ on $K_{i}$. We also need interpolation operators with less regularity requirements than those presented so far. Let for $i \in\{1, \ldots, N+1\}$

$$
F_{i}(v):=\frac{1}{h_{m(i)}} \int_{K_{m(i)}} v, \quad \text { where } \quad m(i):= \begin{cases}i & \text { if } \bar{\kappa}_{h, i} \geqslant \bar{\kappa}_{h, i+1} \\ i+1 & \text { if } \bar{\kappa}_{h, i+1}>\bar{\kappa}_{h, i}\end{cases}
$$

and define for $v \in L^{2}(\Omega)$

$$
Q_{h}^{1} v(x):=F_{i-1}(v) \psi_{i-1}(x)+F_{i}(v) \psi_{i}(x) \quad \text { for } x \in K_{i} .
$$

\subsection{Estimates for the interpolation onto $V_{h}^{1}$}

Theorem 4.1. Let $v \in H^{1}(\Omega)$ and $I_{h}^{1} v \in V_{h}^{1}$ be as in (4.1) with $h \leqslant 1 / 2$. Then, $I_{h}^{1} v$ is well defined if we require that $C_{\mathrm{I}}<\infty, C_{\mathrm{I}}$ from (3.7), and we have the estimate

$$
\left\|v-I_{h}^{1} v\right\|_{L^{2}(\Omega)} \leqslant 2 C_{\mathrm{I}}\left(\sum_{i=1}^{N+1} h_{i}^{2}\|v\|_{\bar{\kappa}_{h, i} ; K_{i}}^{2}\right)^{1 / 2}
$$

while for $v \in H^{2}(\Omega)$

$$
2\left\|h^{-1}\left(v-I_{h}^{1} v\right)\right\|_{L^{2}(\Omega)}+\left\|v-I_{h}^{1} v\right\|_{\bar{\kappa}_{h} ; \Omega} \leqslant 4 C_{\mathrm{I}}\left\|h L\left(\bar{\gamma}_{h}\right) v\right\|_{L^{2}(\Omega)} .
$$

Proof. It is convenient to consider the interval $(0, h)$ in place of $K_{i}$ and some $v \in H^{1}(0, h)$. For this let $\bar{\gamma}:=\bar{\gamma}_{h, i}, \bar{\kappa}:=\bar{\kappa}_{h, i}$ (i. e., $\left.\bar{\gamma}= \pm \bar{\kappa}^{2}\right), \bar{L}:=L(\bar{\gamma})$ and $\bar{G}(.,.) \equiv \bar{G}(., . ; \bar{\gamma})$ be Green's function for the homogeneous Dirichlet boundary value problem for $\bar{L}$ on $(0, h)$ (see Section 4.3). Let $v_{h}:=I_{h}^{1} v$. Since $\left(v-v_{h}\right)(0)=\left(v-v_{h}\right)(h)=0$ and $\bar{L} v_{h}=0$, we get by Green's representation formula and integration by parts

$$
\begin{aligned}
\left(v-v_{h}\right)(x) & =\int_{0}^{h} \bar{G}(x, y) \bar{L}\left(v-v_{h}\right)(y) d y=\int_{0}^{h} \bar{G}(x, y)\left(-v^{\prime \prime}+\bar{\gamma} v\right)(y) d y \\
& =\int_{0}^{h}\left\{\partial_{2} \bar{G}(x, y) v^{\prime}(y)+\bar{\gamma} \bar{G}(x, y) v(y)\right\} d y
\end{aligned}
$$

for all $x \in(0, h)$. This leads to the pointwise inequality

$$
\left|\left(v-v_{h}\right)(x)\right| \leqslant\left\|\partial_{2} \bar{G}(x, .)\right\|_{L^{2}(0, h)}\left\|v^{\prime}\right\|_{L^{2}(0, h)}+\bar{\kappa}^{2}\|\bar{G}(x, .)\|_{L^{2}(0, h)}\|v\|_{L^{2}(0, h)}
$$


and taking the $L^{2}$-norm over $(0, h)$ yields

$$
\left\|v-v_{h}\right\|_{L^{2}(0, h)} \leqslant h^{1 / 2} \sup _{x \in(0, h)}\left\{\left\|\partial_{2} \bar{G}(x, .)\right\|_{L^{2}(0, h)}\left\|v^{\prime}\right\|_{L^{2}(0, h)}+\bar{\kappa}^{2}\|\bar{G}(x, .)\|_{L^{2}(0, h)}\|v\|_{L^{2}(0, h)}\right\} .
$$

Now we use the estimates for Green's function from Theorem 4.4 in the three different cases. Note that $h \leqslant 1 / 2$ implies $\bar{\kappa} \geqslant \pi$ in Cases 2 and 3 .

Case $1 \bar{\gamma} h^{2} \leqslant-(\pi / 2)^{2}$ (i. e., $\left.\bar{\kappa} h \geqslant \pi / 2\right)$. Then

$$
\begin{aligned}
\left\|v-v_{h}\right\|_{L^{2}(0, h)} & \leqslant \frac{h}{|\sin (\bar{\kappa} h)|}\left\|v^{\prime}\right\|_{L^{2}(0, h)}+\frac{\bar{\kappa} h}{|\sin (\bar{\kappa} h)|}\|v\|_{L^{2}(0, h)} \\
& \leqslant \frac{h}{|\sin (\bar{\kappa} h)|}\left(\left\|v^{\prime}\right\|_{L^{2}(0, h)}+\bar{\kappa}\|v\|_{L^{2}(0, h)}\right) \leqslant \frac{2}{|\sin (\bar{\kappa} h)|} h\|v\|_{\bar{\kappa} ;(0, h)} .
\end{aligned}
$$

Case $2|\bar{\gamma}| h^{2}<(\pi / 2)^{2}$ (i. e., $\left.\bar{\kappa} h<\pi / 2\right)$. Then

$$
\left\|v-v_{h}\right\|_{L^{2}(0, h)} \leqslant h\left(\left\|v^{\prime}\right\|_{L^{2}(0, h)}+\bar{\kappa}(\bar{\kappa} h)\|v\|_{L^{2}(0, h)}\right) \leqslant 2 h\|v\|_{\bar{\kappa} ;(0, h)} .
$$

Case $3 \bar{\gamma} h^{2} \geqslant(\pi / 2)^{2}$ (i. e., $\left.\bar{\kappa} h \geqslant \pi / 2\right)$. Then

$$
\begin{aligned}
\left\|v-v_{h}\right\|_{L^{2}(0, h)} & \leqslant \frac{h^{1 / 2}}{\bar{\kappa}^{1 / 2}}\left\|v^{\prime}\right\|_{L^{2}(0, h)}+(\bar{\kappa} h)^{1 / 2}\|v\|_{L^{2}(0, h)} \\
& \leqslant \frac{h^{1 / 2}}{\bar{\kappa}^{1 / 2}}\left(\left\|v^{\prime}\right\|_{L^{2}(0, h)}+\bar{\kappa}\|v\|_{L^{2}(0, h)}\right) \leqslant 2 h\|v\|_{\bar{\kappa} ;(0, h)} .
\end{aligned}
$$

So we can bound $\left\|v-v_{h}\right\|_{L^{2}(0, h)}$ by $2 h /|\sin (\bar{\kappa} h)|\|v\|_{\bar{\kappa} ;(0, h)}$ in Case 1 and by $2 h\|v\|_{\bar{\kappa} ;(0, h)}$ in Cases 2 and 3.

If $\bar{L} v$ exists, we start with the representation

$$
\left(v-v_{h}\right)(x)=\int_{0}^{h} \bar{G}(x, y) \bar{L} v(y) d y
$$

to get

$$
\left\|v-v_{h}\right\|_{L^{2}(0, h)} \leqslant h^{1 / 2} \sup _{x \in(0, h)}\left\{\|\bar{G}(x, .)\|_{L^{2}(0, h)}\right\}\|\bar{L} v\|_{L^{2}(0, h)}
$$

For the factor in front of $\|\bar{L} v\|_{L^{2}(0, h)}$ we get the bounds $h^{1 / 2} h^{1 / 2} /(\bar{\kappa}|\sin (\bar{\kappa} h)|) \leqslant 2 h^{2} /|\sin (\bar{\kappa} h)|$ (Case 1 ), $h^{1 / 2} h^{3 / 2} \leqslant 2 h^{2}$ (Case 2 ), and $h^{1 / 2} / \bar{\kappa}^{3 / 2} \leqslant 2 h^{2}$ (Case 3 ). To derive the result for the energy norm we start from

$$
\bar{\kappa}_{*}\left\|v-v_{h}\right\|_{L^{2}(0, h)} \leqslant \bar{\kappa}_{*} h^{1 / 2} \sup _{x \in(0, h)}\left\{\|\bar{G}(x, .)\|_{L^{2}(0, h)}\right\}\|\bar{L} v\|_{L^{2}(0, h)} .
$$

For the factor in front of $\|\bar{L} v\|_{L^{2}(0, h)}$ we get now the bounds $\bar{\kappa} h^{1 / 2} h^{1 / 2} /(\bar{\kappa}|\sin (\bar{\kappa} h)|) \leqslant$ $2 h /|\sin (\bar{\kappa} h)|($ Case 1$), \bar{\kappa}_{*} h^{1 / 2} h^{3 / 2} \leqslant 2 h$ (Case 2 ), and $\bar{\kappa} h^{1 / 2} / \bar{\kappa}^{3 / 2} \leqslant 2 h$ (Case 3 ).

Finally, to bound derivatives we start from

$$
\left(v-v_{h}\right)^{\prime}(x)=\int_{0}^{h} \partial_{1} \bar{G}(x, y) \bar{L} v(y) d y
$$


to get

$$
\begin{aligned}
\left\|\left(v-v_{h}\right)^{\prime}\right\|_{L^{2}(0, h)}^{2} & =\int_{0}^{h}\left(\int_{0}^{h} \partial_{1} \bar{G}(x, y) \bar{L} v(y) d y\right)^{2} d x \\
& =\int_{0}^{h} \int_{0}^{h}\left(\int_{0}^{h} \partial_{1} \bar{G}(x, y) \partial_{1} \bar{G}(x, z) d x\right) \bar{L} v(y) \bar{L} v(z) d y d z \\
& \leqslant \int_{0}^{h} \int_{0}^{h}\left\|\partial_{1} \bar{G}(., y)\right\|_{L^{2}(0, h)}\left\|\partial_{1} \bar{G}(., z)\right\|_{L^{2}(0, h)}|\bar{L} v(y)||\bar{L} v(z)| d y d z \\
& \leqslant h\left(\sup _{y \in(0, h)}\left\{\left\|\partial_{1} \bar{G}(., y)\right\|_{L^{2}(0, h)}\right\}\|\bar{L} v\|_{L^{2}(0, h)}\right)^{2} .
\end{aligned}
$$

By symmetry of Green's function we have $\left\|\partial_{1} \bar{G}(., y)\right\|_{L^{2}(0, h)}=\left\|\partial_{2} \bar{G}(y, .)\right\|_{L^{2}(0, h)}$ and we can thus refer to the same estimates as above to bound $\left\|\left(v-v_{h}\right)^{\prime}\right\|_{L^{2}(0, h)}$ by $2 h /|\sin (\bar{\kappa} h)|\|\bar{L} v\|_{L^{2}(0, h)}$ in Case 1 and $2 h\|\bar{L} v\|_{L^{2}(0, h)}$ in Cases 2 and 3.

These local estimates can directly be rewritten as estimates on $K_{i}$ and the estimates (4.6)-(4.7) for $v-v_{h}$ on $\Omega$ are obtained from this after squaring and summation over all $K_{i}$.

Theorem 4.2. Let $v \in H^{1}(\Omega)$ and $Q_{h}^{1} v \in V_{h}^{1}$ be as in (4.5) with $h \leqslant 1 / 2$. Then, $Q_{h}^{1} v$ is well defined if we require that $C_{\mathrm{I}}<\infty, C_{\mathrm{I}}$ from (3.7), and we have the estimates

$$
\begin{aligned}
\left\|\bar{\kappa}_{h}\left(v-Q_{h}^{1} v\right)\right\|_{L^{2}(\Omega)} & \leqslant \sqrt{3}(1+\sqrt{2 \sigma}) C_{\mathrm{I}}\left\|\bar{\kappa}_{h} v\right\|_{L^{2}(\Omega)}, \\
\left\|v-Q_{h}^{1} v\right\|_{L^{2}(\Omega)} & \leqslant \sqrt{3}(2+\sqrt{2 \sigma}) C_{\mathrm{I}}\left(\sum_{i=1}^{N+1} h_{i}^{2}\|v\|_{\bar{\kappa}_{h} ; K_{i}}^{2}\right) .
\end{aligned}
$$

Here, $\sigma:=\max _{i=1, \ldots, N}\left\{h_{i} / h_{i+1}, h_{i+1} / h_{i}\right\}$.

Proof. From its definition (4.4) we see $\left|F_{i}(v)\right| \leqslant 1 / h_{m(i)}^{1 / 2}\|v\|_{L^{2}\left(K_{m(i)}\right)}$, and thus

$$
\begin{aligned}
& \left\|Q_{h}^{1} v\right\|_{L^{2}\left(K_{i}\right)} \leqslant\left|F_{i-1}(v)\right|\left\|\psi_{i-1}\right\|_{L^{2}\left(K_{i}\right)}+\left|F_{i}(v)\right|\left\|\psi_{i}\right\|_{L^{2}\left(K_{i}\right)} \\
& \quad \leqslant\left(h_{m(i-1)}\left|F_{i-1}(v)\right|^{2}+h_{m(i)}\left|F_{i}(v)\right|^{2}\right)^{1 / 2}\left(\frac{1}{h_{m(i-1)}}\left\|\psi_{i-1}\right\|_{L^{2}\left(K_{i}\right)}^{2}+\frac{1}{h_{m(i)}}\left\|\psi_{i}\right\|_{L^{2}\left(K_{i}\right)}^{2}\right)^{1 / 2} \\
& \quad \leqslant C_{\mathrm{I}}\left(\|v\|_{L^{2}\left(K_{m(i-1)}\right)}^{2}+\|v\|_{L^{2}\left(K_{m(i)}\right)}^{2}\right)^{1 / 2}\left(\frac{h_{i}}{h_{m(i-1)}}+\frac{h_{i}}{h_{m(i)}}\right)^{1 / 2} \\
& \quad \leqslant \sqrt{2 \sigma} C_{\mathrm{I}}\|v\|_{L^{2}\left(K_{m(i-1)} \cup K_{m(i)}\right)} .
\end{aligned}
$$

By definition of $m(i-1), m(i)$ it holds

$$
\bar{\kappa}_{h, i}\|v\|_{L^{2}\left(K_{m(i-1)} \cup K_{m(i)}\right)} \leqslant\left\|\bar{\kappa}_{h} v\right\|_{L^{2}\left(K_{m(i-1)} \cup K_{m(i)}\right)} \leqslant\left\|\bar{\kappa}_{h} v\right\|_{L^{2}\left(K_{i-1} \cup K_{i} \cup K_{i+1}\right)}
$$

and therefore

$$
\bar{\kappa}_{h, i}\left\|v-v_{h}\right\|_{L^{2}\left(K_{i}\right)} \leqslant\left\|\bar{\kappa}_{h} v\right\|_{L^{2}\left(K_{i}\right)}+\bar{\kappa}_{h, i}\left\|v_{h}\right\|_{L^{2}\left(K_{i}\right)} \leqslant(1+\sqrt{2 \sigma}) C_{\mathrm{I}}\left\|\bar{\kappa}_{h} v\right\|_{L^{2}\left(K_{i-1} \cup K_{i} \cup K_{i+1}\right)} .
$$

To prove the next assertion we start from the decomposition

$$
v-Q_{h}^{1} v=v-I_{h}^{1} v+\left(v\left(x_{i-1}\right)-F_{i-1}(v)\right) \psi_{i-1}+\left(v\left(x_{i}\right)-F_{i}(v)\right) \psi_{i},
$$


which can be estimated using previous results by

$$
\begin{aligned}
\| v- & Q_{h}^{1} v \|_{L^{2}\left(K_{i}\right)} \\
& =\left\|v-I_{h}^{1} v\right\|_{L^{2}\left(K_{i}\right)}+h_{m(i-1)}^{1 / 2}\left\|v^{\prime}\right\|_{L^{2}\left(K_{m(i-1)}\right)}\left\|\psi_{i-1}\right\|_{L^{2}\left(K_{i}\right)}+h_{m(i)}^{1 / 2}\left\|v^{\prime}\right\|_{L^{2}\left(K_{m(i)}\right)}\left\|\psi_{i}\right\|_{L^{2}\left(K_{i}\right)} \\
& \leqslant 2 C_{\mathrm{I}} h_{i}\|v\|_{\bar{\kappa}_{h, i} ; K_{i}}+\sqrt{2 \sigma} C_{\mathrm{I}}\left\|h v^{\prime}\right\|_{L^{2}\left(K_{i-1} \cup K_{i} \cup K_{i+1}\right)} .
\end{aligned}
$$

The final result for the $L^{2}$-norms follows by summation over $i$.

\subsection{Estimates for the interpolation onto $V_{h}^{2}$}

Theorem 4.3. Let $v \in H^{1}(\Omega)$ and $I_{h}^{2} v, \widetilde{I}_{h}^{2} v \in V_{h}^{2}$ be as in (4.2) and (4.3), respectively, with $h \leqslant 1 / 2$. Then, both interpolants are well defined if we require that $C_{\mathrm{I}}<\infty, C_{\mathrm{I}}$ from (3.7), and we have the estimate

$$
\left\|v-I_{h}^{2} v\right\|_{L^{2}(\Omega)} \leqslant 2 C_{\mathrm{I}}\left(\sum_{i=1}^{N+1} h_{i}^{2}\left\|v^{\prime}\right\|_{L^{2}\left(K_{i}\right)}^{2}\right)^{1 / 2} .
$$

The estimate (4.7) holds correspondingly with $\widetilde{I}_{h}^{2}$ for $v \in H^{2}(\Omega)$ and, furthermore, for $v \in$ $H^{3}(\Omega)$

$$
2\left\|h^{-1}\left(v-\widetilde{I}_{h}^{2} v\right)\right\|_{L^{2}(\Omega)}+\left\|v-\widetilde{I}_{h}^{2} v\right\|_{\bar{\kappa}_{h} ; \Omega} \leqslant 4 C_{\mathrm{I}}\left\|h^{2}\left(L\left(\bar{\gamma}_{h}\right) v\right)_{h}^{\prime}\right\|_{L^{2}(\Omega)} .
$$

$(.)_{h}^{\prime}$ denotes the piecewise derivative with respect to $\mathcal{K}$.

Proof. We use the notation introduced in the proof of Theorem 4.1. From definition (4.2) we obtain

$$
\begin{aligned}
\left(v-I_{h}^{2} v\right)(x) & =v(x)-\left(v(0) \psi_{0}(x)+v(h) \psi_{1}(x)+\frac{\bar{\gamma}}{2}(v(0)+v(h)) \psi_{1 / 2}(x)\right) \\
& =v(x)-\left(v(0) \psi_{0}(x)+v(h) \psi_{1}(x)+\frac{1}{2}(v(0)+v(h))\left(1-\psi_{0}(x)-\psi_{1}(x)\right)\right) \\
& =v(x)-\frac{1}{2}(v(0)+v(h))-\frac{1}{2}(v(h)-v(0))\left(\psi_{1}(x)-\psi_{0}(x)\right)
\end{aligned}
$$

and this yields the pointwise bound

$$
\left|\left(v-I_{h}^{2} v\right)(x)\right| \leqslant\left(1+\frac{1}{2}\left\|\psi_{0}\right\|_{L^{\infty}(0, h)}+\frac{1}{2}\left\|\psi_{1}\right\|_{L^{\infty}(0, h)}\right) \int_{0}^{h}\left|v^{\prime}(y)\right| d y
$$

For $\bar{\gamma}>0$, or $\bar{\gamma}<0$ and $\bar{\kappa} h<\pi / 2$, we have $\left\|\psi_{0}\right\|_{L^{\infty}(0, h)}=\left\|\psi_{1}\right\|_{L^{\infty}(0, h)}=1$ and therefore

$$
\left\|v-I_{h}^{2} v\right\|_{L^{2}(0, h)} \leqslant 2 h\left\|v^{\prime}\right\|_{L^{2}(0, h)},
$$

while for $\bar{\gamma}<0$ and $\bar{\kappa} h \geqslant \pi / 2$ we obtain

$$
\left\|v-I_{h}^{2} v\right\|_{L^{2}(0, h)} \leqslant \frac{2 h}{|\sin (\bar{\kappa} h)|}\left\|v^{\prime}\right\|_{L^{2}(0, h)} .
$$

This can directly be rewritten as an estimate for $v-v_{h}$ on $K_{i}$ and the estimate (4.10) for $v-v_{h}$ on $\Omega$ is obtained from this after squaring and summation over all $K_{i}$. 
We let $v_{h}:=\widetilde{I}_{h}^{2} v$ and get by the representation formula (with $\bar{G}(x, y)=G(x, y ; \bar{\gamma})$ )

$$
\left(v-v_{h}\right)(x)=\int_{0}^{h} \bar{G}(x, y) \bar{L}\left(v-v_{h}\right)(y) d y=\int_{0}^{h} \bar{G}(x, y)\left(\bar{L} v(y)-\frac{1}{h} \int_{0}^{h} \bar{L} v\right) d y .
$$

If we only exploit the $L^{2}$-stability of the mean value, we can repeat the arguments from Theorem 4.1 to get the same results as for $I_{h}^{1}$. Now let $v \in H^{3}(\Omega)$. Using $\left\|\bar{L} v-\int_{0}^{h} \bar{L} v / h\right\|_{L^{2}(0, h)} \leqslant$ $h\left\|(\bar{L} v)^{\prime}\right\|_{L^{2}(0, h)}$, we can achieve the pointwise bound

$$
\left|\left(v-v_{h}\right)(x)\right| \leqslant h^{3 / 2} \sup _{x \in(0, h)}\left\{\|\bar{G}(x, .)\|_{L^{2}(0, h)}\right\}\left\|(\bar{L} v)^{\prime}\right\|_{L^{2}(0, h)} .
$$

Similarly, we get the pointwise estimate for $\left(v-v_{h}\right)^{\prime}(x)$ with $\partial_{1} \bar{G}$ on the left-hand side and the remainder is as in the proof of Theorem 4.1.

\subsection{Estimates for Green's functions}

The boundary value problem (2.1)-(2.2) has a Green's function $G: \Omega \times \Omega \rightarrow \mathbb{R}$, which allows the solution to be represented as

$$
u(x)=\int_{0}^{1} G(x, y) L u(y) d y=\int_{0}^{1} G(x, y) f(y) d y
$$

for every $f \in L^{1}(\Omega)$. To construct $G$ one can determine the fundamental system $\left\{\Psi_{0}, \Psi_{1}\right\}$ that fulfills the boundary value problems $L(\gamma) \Psi_{0}=0$ in $\Omega, \Psi_{0}(0)=1, \Psi_{0}(1)=0$, and $L(\gamma) \Psi_{1}=0$ in $\Omega, \Psi_{1}(0)=0, \Psi_{1}(1)=1$. Then $G$ is given by

$$
G(x, y)=\frac{1}{\Psi_{0}^{\prime}(0)} \begin{cases}\Psi_{0}(x) \Psi_{1}(y) & \text { for } y \leqslant x \\ \Psi_{1}(x) \Psi_{0}(y) & \text { for } x \leqslant y\end{cases}
$$

$G$ is explicitly known only in very special cases, for example, when $\gamma$ is constant.

The results of the previous section required estimates on Green's function $G(., . ; \bar{\gamma})$ for the boundary value problem (2.1)-(2.2) with constant coefficient $\bar{\gamma}$ on $(0, h)$. The following theorem provides these estimates (some details were elaborated in the diploma thesis of A. Shutovich that has been prepared under the supervision of the author).

Theorem 4.4. Let $G$ be Green's function of the boundary value problem (2.1)-(2.2) with constant coefficient $\bar{\gamma}= \pm \bar{\kappa}^{2}$ on $(0, h)$, then

$$
\sup _{x \in(0, h)}\left\{\|G(x, . ; \bar{\gamma})\|_{L^{2}(0, h)}\right\} \leqslant h^{3 / 2} \begin{cases}\frac{1}{\bar{\kappa} h|\sin (\bar{\kappa} h)|} & \text { if } \bar{\gamma}<0, \bar{\kappa} h>\pi / 2, \\ \frac{1}{12} & \text { if } \bar{\kappa} h<\pi / 2 \\ \frac{1}{(\bar{\kappa} h)^{3 / 2}} & \text { if } \bar{\gamma}>0, \bar{\kappa} h>\pi / 2\end{cases}
$$

and

$$
\sup _{x \in(0, h)}\left\{\left\|\partial_{2} G(x, . ; \bar{\gamma})\right\|_{L^{2}(0, h)}\right\} \leqslant h^{1 / 2} \begin{cases}\frac{1}{|\sin (\bar{\kappa} h)|} & \text { if } \bar{\gamma}<0, \bar{\kappa} h>\pi / 2 \\ 1 & \text { if } \bar{\kappa} h<\pi / 2 \\ \frac{1}{(\bar{\kappa} h)^{1 / 2}} & \text { if } \bar{\gamma}>0, \bar{\kappa} h>\pi / 2 .\end{cases}
$$


Proof. If $G$ is Green's function of the boundary value problem (2.1)-(2.2) with constant coefficient on $(0, h)$, then $\widehat{G}(\xi, \eta ; z)=1 / h G\left(h \xi, h \eta ; z / h^{2}\right)$ is the corresponding Green's function on $(0,1)$. Therefore, we obtain after scaling

$$
\begin{aligned}
\|G(x, . ; \bar{\gamma})\|_{L^{2}(0, h)} & =h^{3 / 2}\left\|\widehat{G}\left(x / h, . ; \bar{\gamma} h^{2}\right)\right\|_{L^{2}(0,1)}, \\
\left\|\partial_{2} G(x, . ; \bar{\gamma})\right\|_{L^{2}(0, h)} & =h^{1 / 2}\left\|\partial_{2} \widehat{G}\left(x / h, . ; \bar{\gamma} h^{2}\right)\right\|_{L^{2}(0,1)}
\end{aligned}
$$

In the following, we will estimate

$$
g_{0}(\xi ; z):=\|\widehat{G}(\xi, . ; z)\|_{L^{2}(0,1)}^{2} \quad \text { and } \quad g_{1}(\xi ; z):=\left\|\partial_{2} \widehat{G}(\xi, . ; z)\right\|_{L^{2}(0,1)}^{2}
$$

for different values of $z$.

Case 1: $z<0, k:=\sqrt{|z|} \geqslant \pi / 2, \sin (k) \neq 0 \quad$ We start calculating $g_{0}\left(\xi ;-k^{2}\right)$ to

$$
g_{0}\left(\xi ;-k^{2}\right)=-\frac{\sin (k \xi) \sin (k(1-\xi))}{2 k^{3}|\sin (k)|}+\frac{(1-\xi) \sin (k \xi)^{2}+\xi \sin (k(1-\xi))^{2}}{2 k^{2} \sin (k)^{2}} .
$$

Since $\xi \in(0,1),(1-\xi) \sin (k \xi)^{2}+\xi \sin (k(1-\xi))^{2} \leqslant(1-\xi)+\xi=1$, and $\sin (k) / k \leqslant 1$, one gets that

$$
g_{0}\left(\xi ;-k^{2}\right) \leqslant \frac{1}{2 k^{3}|\sin (k)|}+\frac{1}{2 k^{2} \sin (k)^{2}} \leqslant \frac{1}{k^{2} \sin (k)^{2}}
$$

thus

$$
\sup _{\xi \in(0,1)}\left\{\left\|\widehat{G}\left(\xi, . ;-k^{2}\right)\right\|_{L^{2}(0,1)}\right\} \leqslant \frac{1}{k|\sin (k)|}
$$

Similarly, we get for $g_{1}\left(\xi ;-k^{2}\right)$

$$
\begin{aligned}
g_{1}\left(\xi ;-k^{2}\right) & =\frac{\sin (k \xi) \sin (k(1-\xi))}{2 k|\sin (k)|}+\frac{(1-\xi) \sin (k \xi)^{2}+\xi \sin (k(1-\xi))^{2}}{2 \sin (k)^{2}} \\
& \leqslant \frac{1}{2 k|\sin (k)|}+\frac{1}{2 \sin (k)^{2}} \leqslant \frac{1}{\sin (k)^{2}}
\end{aligned}
$$

resulting in

$$
\sup _{\xi \in(0,1)}\left\{\left\|\partial_{2} \widehat{G}\left(\xi, . ;-k^{2}\right)\right\|_{L^{2}(0,1)}\right\} \leqslant \frac{1}{|\sin (k)|} .
$$

Case 2: $z>0, k:=\sqrt{|z|} \geqslant \pi / 2$ We now get

$$
\begin{aligned}
g_{0}\left(\xi ; k^{2}\right) & =\frac{\sinh (k \xi) \sinh (k(1-\xi))}{2 k^{3} \sinh (k)}-\frac{(1-\xi) \sinh (k \xi)^{2}+\xi \sinh (k(1-\xi))^{2}}{2 k^{2} \sinh (k)^{2}} \\
& =: T_{1}-T_{2} .
\end{aligned}
$$

Since all terms $g_{0}, T_{1}, T_{2}$ are positive, we conclude $T_{2} \leqslant T_{1}$ and $g_{0}\left(\xi ; k^{2}\right) \leqslant T_{1}$. By elementary calculus we see that $e^{k} / 4 \leqslant\left(e^{k}-e^{-k}\right) / 2$ for $k \geqslant \log (2) / 2$ and thus

$$
T_{1} \leqslant \frac{1}{4} \frac{e^{k}}{2 k^{3} \sinh (k)} \leqslant \frac{1}{2} \frac{\sinh (k)}{2 k^{3} \sinh (k)} \leqslant \frac{1}{4 k^{3}}
$$


showing

$$
\sup _{\xi \in(0,1)}\left\{\left\|\widehat{G}\left(\xi, . ; k^{2}\right)\right\|_{L^{2}(0,1)}\right\} \leqslant \frac{1}{2 k^{3 / 2}}
$$

For $g_{1}\left(\xi ; k^{2}\right)$ we get

$$
g_{1}\left(\xi ; k^{2}\right)=k^{2}\left(T_{1}+T_{2}\right) \leqslant 2 k^{2} T_{1} \leqslant \frac{1}{2 k}
$$

and so

$$
\sup _{\xi \in(0,1)}\left\{\left\|\partial_{2} \widehat{G}\left(\xi, . ; k^{2}\right)\right\|_{L^{2}(0,1)}\right\} \leqslant \frac{1}{k^{1 / 2}}
$$

Case 3: $z<0, k:=\sqrt{|z|}<\pi / 2$ Taking the derivative of $g_{0}\left(. ;-k^{2}\right)$ (given above) we arrive at

$$
g_{0}^{\prime}\left(\xi ;-k^{2}\right)=\frac{\xi(1-\xi)}{\sin (k)^{2}}\left(\frac{\sin (2 k \xi)}{2 k \xi}-\frac{\sin (2 k(1-\xi))}{2 k(1-\xi)}\right) .
$$

By monotonicity of $t \mapsto \sin (t) / t$ in $(0, \pi)$ we see that $g_{0}^{\prime}\left(. ;-k^{2}\right)$ is positive for $\xi \in(0,1 / 2)$ and negative for $\xi \in(1 / 2,1)$. Thus the maximum of $g_{0}\left(. ;-k^{2}\right)$ is attained for $\xi=1 / 2$. Using $\sin (t) \geqslant t-t^{3} / 6$ we obtain

$$
\sup _{\xi \in(0,1)}\left\{\left\|\widehat{G}\left(\xi, . ;-k^{2}\right)\right\|_{L^{2}(0,1)}\right\} \leqslant g_{0}\left(\frac{1}{2} ; k^{2}\right)=\frac{k-\sin (k)}{2 k^{3}} \frac{\sin (k / 2)^{2}}{\sin (k)^{2}} \leqslant \frac{k^{3} / 6}{2 k^{3}}=\frac{1}{12} .
$$

For $g_{1}\left(. ;-k^{2}\right)$ we get

$$
g_{1}^{\prime}\left(\xi ;-k^{2}\right)=\frac{\cos (2 k \xi)-\cos (2 k(1-\xi))+k((1-\xi) \sin (2 k \xi)-\xi \sin (2 k(1-\xi)))}{1-\cos (2 k)} .
$$

As before (using monotonicity of the mappings $\xi \mapsto \cos (2 k \xi)-\cos (2 k(1-\xi))$ and $\xi \mapsto$ $(1-\xi) k \sin (2 k \xi)-\xi k \sin (2 k(1-\xi)))$ we conclude that $g_{1}\left(. ;-k^{2}\right)$ attains its maximum at $\xi=1 / 2$ and this gives

$$
\sup _{\xi \in(0,1)}\left\{\left\|\partial_{2} \widehat{G}\left(\xi, . ;-k^{2}\right)\right\|_{L^{2}(0,1)}\right\} \leqslant g_{1}\left(\frac{1}{2}\right)=\frac{k+\sin (k)}{2 k} \frac{\sin (k / 2)^{2}}{\sin (k)^{2}} \leqslant \frac{2 k}{2 k}=1 .
$$

Case 4: $z>0, k:=\sqrt{|z|}<\pi / 2$ As above we see from

$$
g_{0}^{\prime}\left(\xi ; k^{2}\right)=\frac{\xi(1-\xi)}{\sinh (k)^{2}}\left(\frac{\sinh (2 k(1-\xi))}{2 k(1-\xi)}-\frac{\sinh (2 k \xi)}{2 k \xi}\right)
$$

and monotonicity of $t \mapsto \sinh (t) / t$ that the maximum of $g_{0}\left(. ; k^{2}\right)$ is attained for $\xi=1 / 2$. Using trigonometric relations and $\sinh (t) \leqslant t+t^{3} / 6 \cosh (t)$ we obtain

$$
\begin{aligned}
\sup _{\xi \in(0,1)}\left\{\left\|\widehat{G}\left(\xi, . ; k^{2}\right)\right\|_{L^{2}(0,1)}\right\} & \leqslant g_{0}\left(\frac{1}{2} ; k^{2}\right)=\frac{\sinh (k)-k}{2 k^{3}} \frac{\sinh (k / 2)^{2}}{\sinh (k)^{2}}=\frac{\sinh (k)-k}{4 k^{3}(1+\cosh (k))} \\
& \leqslant \frac{k^{3} / 6 \cosh (k)}{4 k^{3}(1+\cosh (k))} \leqslant \frac{1}{24} .
\end{aligned}
$$


For $g_{1}\left(. ; k^{2}\right)$ we get

$$
g_{1}^{\prime}\left(\xi ; k^{2}\right)=\frac{\cosh (2 k \xi)-\cosh (2 k(1-\xi))-k((1-\xi) \sinh (2 k \xi)-\xi \sinh (2 k(1-\xi)))}{1-\cosh (2 k)}
$$

and the reasoning is similar to the one used before to conclude that $g_{1}\left(. ; k^{2}\right)$ is maximal at $\xi=1 / 2$. Using $\sinh (t) \leqslant t \cosh (t)$ we obtain

$$
\begin{aligned}
\sup _{\xi \in(0,1)}\left\{\left\|\partial_{2} \widehat{G}\left(\xi, . ; k^{2}\right)\right\|_{L^{2}(0,1)}\right\} & \leqslant g_{1}\left(\frac{1}{2}, k^{2}\right)=\frac{k+\sinh (k)}{2 k} \frac{\sinh (k / 2)^{2}}{\sinh (k)^{2}}=\frac{k+\sinh (k)}{2 k(1+\cosh (k))} \\
& \leqslant \frac{k}{2 k}=\frac{1}{2} .
\end{aligned}
$$

\section{A posteriori error estimates}

\subsection{Error representation}

Using the solution properties of $u \in V$ and $u_{h} \in V_{h} \subset V$, respectively, we get, for arbitrary $v \in V$ and $v_{h} \in V_{h}$, the representation

$$
\begin{aligned}
B(u & \left.-u_{h}, v\right)=\int_{0}^{1}\left\{\left(u-u_{h}\right)^{\prime} v^{\prime}+\gamma\left(u-u_{h}\right) v\right\} \\
& =\sum_{i=1}^{N}\left[u_{h}^{\prime}\right]_{x_{i}}\left(v-v_{h}\right)\left(x_{i}\right)-\int_{0}^{1} r_{h}\left(v-v_{h}\right)+\int_{0}^{1}\left\{\left(f-f_{h}\right) v-\left(\gamma-\gamma_{h}\right) u_{h} v\right\}
\end{aligned}
$$

for the error $u-u_{h}$ with the derivative jump in the grid point $x_{i}$

$$
\left[u_{h}^{\prime}\right]_{x_{i}}:=\lim _{s \rightarrow 0}\left(u_{h}^{\prime}\left(x_{i}+s\right)-u_{h}^{\prime}\left(x_{i}-s\right)\right)
$$

and the residual $r_{h}$ piecewise defined by

$$
r_{h}:=-u_{h}^{\prime \prime}+\gamma_{h} u_{h}-f_{h} \quad \text { on } K
$$

for all $K \subset \mathcal{K}$. For $u_{h} \in V_{h}^{1}$ the residual is $r_{h}=L\left(\gamma_{h}\right) u_{h}-f_{h}=L\left(\bar{\gamma}_{h}\right) u_{h}+\left(\gamma_{h}-\bar{\gamma}_{h}\right) u_{h}-$ $f_{h}=\left(\gamma_{h}-\bar{\gamma}_{h}\right) u_{h}-f_{h}$. The choice $\gamma_{h}=\bar{\gamma}_{h}$ then gives $r_{h}=-f_{h}$. In case $u_{h} \in V_{h}^{2}$ the residual is $r_{h}=L\left(\bar{\gamma}_{h}\right) u_{h}+\left(\gamma_{h}-\bar{\gamma}_{h}\right) u_{h}-f_{h}$. Note that $L\left(\bar{\gamma}_{h}\right) u_{h}=b_{h}$, where $b_{h}=b_{j}$ on $K=K_{j}$ (see (3.6)) is directly accessible from the discrete solution. In general we thus have $r_{h}=b_{h}-f_{h}+\left(\gamma_{h}-\bar{\gamma}_{h}\right) u_{h}$. If we take $\gamma_{h}=\bar{\gamma}_{h}$, then we find (see Sect. 3.4) $b_{j}=\int_{K_{j}} f_{h} \psi_{j-1 / 2} / \int_{K_{j}} \psi_{j-1 / 2}$ and therefore we get $r_{h}=0$ for piecewise constant $f_{h}$.

For the following section recall the definitions of $c_{\gamma}$ (cf. 2.4), $\Gamma$ (see Section 3.1), $m(i)$ (from (4.4)), and $\sigma$ (Theorem 4.2).

\subsection{Upper bound}

Theorem 5.1. Let $u \in V$ be a solution to (2.1)-(2.2) and $u_{h}$, either in $V_{h}^{1}$ or $V_{h}^{2}$, be the respective solution of the discrete problem (3.1) on a grid with $C_{\mathrm{I}}<\infty, C_{\mathrm{I}}$ from (3.7). Then 
the following a posteriori error estimate is valid

$$
\begin{aligned}
c_{\gamma}\left\|u-u_{h}\right\|_{\kappa ; \Omega} \leqslant 4(1 & +\sqrt{\sigma}) C_{\mathrm{I}}\left(1+\Gamma\left(\kappa, \bar{\kappa}_{h}\right)\right)\left(\sum_{i=1}^{N+1} \eta_{i}^{2}\right)^{1 / 2} \\
& +\left\|\frac{1}{\kappa_{*}}\left(f-f_{h}\right)\right\|_{L^{2}(\Omega)}+\left\|\frac{\gamma-\gamma_{h}}{\gamma_{*}} \kappa_{*} u_{h}\right\|_{L^{2}(\Omega)}
\end{aligned}
$$

with the local error indicator

$$
\eta_{i}:=\left(h_{m(i)} \min \left\{1, \frac{1}{\bar{\kappa}_{h, m(i)} h_{m(i)}}\right\}\left[u_{h}^{\prime}\right]_{x_{i}}^{2}+h_{i}^{2} \min \left\{1, \frac{1}{\bar{\kappa}_{h, i} h_{i}}\right\}^{2}\left\|r_{h}\right\|_{L^{2}\left(K_{i}\right)}^{2}\right)^{1 / 2} .
$$

Proof. From (5.1) we get for arbitrary $v \in V, v_{h} \in V_{h}$,

$$
\begin{aligned}
\left|B\left(u-u_{h}, v\right)\right| \leqslant & \sum_{i=1}^{N}\left|\left[u_{h}^{\prime}\right]_{x_{i}}\left(v-v_{h}\right)\left(x_{i}\right)\right|+\left|\int_{\Omega} r_{h}\left(v-v_{h}\right)\right| \\
& +\left(\left\|\frac{1}{\kappa_{*}}\left(f-f_{h}\right)\right\|_{L^{2}(\Omega)}+\left\|\frac{1}{\kappa_{*}}\left(\gamma-\gamma_{h}\right) u_{h}\right\|_{L^{2}(\Omega)}\right)\left\|\kappa_{*} v\right\|_{L^{2}(\Omega)} .
\end{aligned}
$$

Taking $I_{h}^{1} v$ for $v_{h}$ we would have $\left(v-I_{h}^{1} v\right)\left(x_{i}\right)=0$ and the first term on the right-hand side would disappear. However, if $\bar{\kappa}_{h, i} h_{i}$ is large, we need a better estimate. This can only be achieved taking $v_{h}=Q_{h}^{1} v$, since $Q_{h}^{1}$ is continuous on $L^{2}$ (unlike $I_{h}^{1}$ ). By Theorem 4.2 (proof) we observe

$$
\bar{\kappa}_{h, i}\left\|v-v_{h}\right\|_{L^{2}\left(K_{i}\right)} \leqslant(1+\sqrt{2 \sigma}) C_{\mathrm{I}}\left\|\bar{\kappa}_{h} v\right\|_{L^{2}\left(K_{i-1} \cup K_{i} \cup K_{i+1}\right)},
$$

while for more regular $v$ one can derive

$$
\left\|v-v_{h}\right\|_{L^{2}\left(K_{i}\right)} \leqslant(2+\sqrt{2 \sigma}) C_{\mathrm{I}} h_{i}\|v\|_{\bar{\kappa}_{h} ; K_{i-1} \cup K_{i} \cup K_{i+1}} .
$$

We are now in the position to choose the best of both choices to get

$$
\left\|v-v_{h}\right\|_{L^{2}\left(K_{i}\right)} \leqslant(2+\sqrt{2 \sigma}) C_{\mathrm{I}} h_{i} \min \left\{1, \frac{1}{\bar{\kappa}_{h, i} h_{i}}\right\}\|v\|_{\bar{\kappa}_{h} ; K_{i-1} \cup K_{i} \cup K_{i+1}} .
$$

Thus the residual term is bounded as follows

$$
\begin{aligned}
\left|\int_{\Omega} r_{h}\left(v-v_{h}\right)\right| & \leqslant \sum_{i=1}^{N+1}\left\|r_{h}\right\|_{L^{2}\left(K_{i}\right)}\left\|v-v_{h}\right\|_{L^{2}\left(K_{i}\right)} \\
& \leqslant(2+\sqrt{2 \sigma}) C_{\mathrm{I}} \sum_{i=1}^{N+1} h_{i} \min \left\{1, \frac{1}{\bar{\kappa}_{h, i} h_{i}}\right\}\left\|r_{h}\right\|_{L^{2}\left(K_{i}\right)}\|v\|_{\bar{\kappa}_{h} ; K_{i-1} \cup K_{i} \cup K_{i+1}} \\
& \leqslant \sqrt{3}(2+\sqrt{2 \sigma}) C_{\mathrm{I}}\left(\sum_{i=1}^{N+1} h_{i}^{2} \min \left\{1, \frac{1}{\bar{\kappa}_{h, i} h_{i}}\right\}^{2}\left\|r_{h}\right\|_{L^{2}\left(K_{i}\right)}^{2}\right)^{1 / 2}\|v\|_{\bar{\kappa}_{h}} .
\end{aligned}
$$

In order to estimate the jump residual term, we first observe

$$
\left|\left(v-v_{h}\right)\left(x_{i}\right)\right|=\left|v\left(x_{i}\right)-F_{i}(v)\right|=\left|\frac{1}{h_{m(i)}} \int_{K_{m(i)}}\left\{v\left(x_{i}\right)-v\right\}\right| \leqslant\left\|\sqrt{h} v^{\prime}\right\|_{L^{2}\left(K_{m(i)}\right)} .
$$


A different estimate is provided as follows: first note that

$$
\left|\left(v-v_{h}\right)\left(x_{i}\right)\right| \leqslant\left|v\left(x_{i}\right)\right|+\left|F_{i}(v)\right| \leqslant 2\|v\|_{L^{\infty}\left(K_{m(i)}\right)} .
$$

For arbitrary $\varepsilon \in(0,1)$ we have from Lemma 5.1 by rescaling and with $j:=m(i)$

$$
\begin{aligned}
\|v\|_{L^{\infty}\left(K_{j}\right)} & \leqslant \varepsilon h_{j}^{1 / 2}\left\|v^{\prime}\right\|_{L^{2}\left(K_{j}\right)}+\frac{2}{\varepsilon h_{j}^{1 / 2}}\|v\|_{L^{2}\left(K_{j}\right)} \\
& =\varepsilon h_{j}^{1 / 2}\left\|v^{\prime}\right\|_{L^{2}\left(K_{j}\right)}+\frac{2}{\varepsilon h_{j}^{1 / 2} \bar{\kappa}_{h, j}}\left\|\bar{\kappa}_{h, j} v\right\|_{L^{2}\left(K_{j}\right)} \\
& \leqslant\left(\varepsilon^{2} h_{j}+\frac{4}{\varepsilon^{2} h_{j} \bar{\kappa}_{h, j}^{2}}\right)^{1 / 2}\|v\|_{\bar{\kappa}_{h, j} ; K_{j}} .
\end{aligned}
$$

The choice $\varepsilon^{2} h_{j}=2 / \bar{\kappa}_{h, j}$ yields

$$
\|v\|_{L^{\infty}\left(K_{j}\right)} \leqslant \frac{2}{\bar{\kappa}_{h, j}^{1 / 2}}\|v\|_{\bar{\kappa}_{h, j} ; K_{j}}
$$

so that we get

$$
\left|\left(v-v_{h}\right)\left(x_{i}\right)\right| \leqslant \frac{4}{\bar{\kappa}_{h, j}^{1 / 2}}\|v\|_{\bar{\kappa}_{h} ; K_{j}} .
$$

We can take the best of the two choices to conclude

$$
\begin{aligned}
\left|\left(v-v_{h}\right)\left(x_{i}\right)\right| & \leqslant \min \left\{h_{j}^{1 / 2}, \frac{4}{\bar{\kappa}_{h, j}^{1 / 2}}\right\}\|v\|_{\bar{\kappa}_{h} ; K_{j}} \\
& \leqslant 4 h_{j}^{1 / 2} \min \left\{1, \frac{1}{\left(\bar{\kappa}_{h, j} h_{j}\right)^{1 / 2}}\right\}\|v\|_{\bar{\kappa}_{h} ; K_{j}} .
\end{aligned}
$$

This allows us to derive

$$
\begin{aligned}
\sum_{i=1}^{N}\left|\left[u_{h}^{\prime}\right]_{x_{i}}\right|\left|\left(v-v_{h}\right)\left(x_{i}\right)\right| & \leqslant 4 \sum_{i=1}^{N} h_{m(i)}^{1 / 2} \min \left\{1, \frac{1}{\bar{\kappa}_{h, m(i)} h_{m(i)}}\right\}^{1 / 2}\left|\left[u_{h}^{\prime}\right]_{x_{i}}\right|\|v\|_{\bar{\kappa}_{h, m(i)} ; K_{m(i)}} \\
& \leqslant 8\left(\sum_{i=1}^{N} h_{m(i)} \min \left\{1, \frac{1}{\bar{\kappa}_{h, m(i)} h_{m(i)}}\right\}\left[u_{h}^{\prime}\right]_{x_{i}}^{2}\right)^{1 / 2}\|v\|_{\bar{\kappa}_{h}} .
\end{aligned}
$$

The assertion follows with

$$
\left\|\bar{\kappa}_{h} v\right\|_{L^{2}(\Omega)} \leqslant\|\kappa v\|_{L^{2}(\Omega)}+\left\|\left(\bar{\kappa}_{h}-\kappa\right) v\right\|_{L^{2}(\Omega)} \leqslant\left(1+\Gamma\left(\kappa, \bar{\kappa}_{h}\right)\right)\left\|\kappa_{*} v\right\|_{L^{2}(\Omega)}
$$

and using the inf-sup condition (2.4). Note, that it suffices to consider the interpolation $Q_{h}^{1}$ since $V_{h}^{1} \subset V_{h}^{2}$.

Lemma 5.1. Let $I:=(0,1)$. Then for all $\epsilon>0$ and all $v \in H^{1}(I)$ it holds that

$$
\|v\|_{L^{\infty}(I)} \leqslant \epsilon\left\|v^{\prime}\right\|_{L^{2}(I)}+\frac{3 / 2}{\epsilon}\|v\|_{L^{2}(I)} .
$$


Proof. Let $v \in C^{1}(I), J$ a subinterval in $I$, and $x, y \in J$. Then $v(x)=v(y)+\int_{y}^{x} v^{\prime}$ and this yields

$$
|v(x)|^{2} \leqslant \frac{3}{2}\left(|v(y)|^{2}+2|x-y| \int_{J}\left|v^{\prime}\right|^{2}\right) .
$$

Now we integrate over $y \in J$ to get that for all $x \in J$

$$
|v(x)|^{2} \leqslant \frac{3}{2}\left(\frac{1}{|J|} \int_{J}|v|^{2}+|J| \int_{J}\left|v^{\prime}\right|^{2}\right)
$$

Now choose $x_{0}$ where the maximum of $v$ is attained, choose $\epsilon>0$ and take $J$ as an interval of length $|J|=2 \epsilon^{2} / 3$ around $x_{0}$. Taking the root and a density argument proves the assertion.

\subsection{Lower bound}

Theorem 5.2. Let $u \in V$ be a solution to (2.1)-(2.2) and $u_{h} \in V_{h}^{1}$ be the solution of the discrete problem (3.1) with $\gamma_{h}=\bar{\gamma}_{h}$ on a suitable grid. Then the lower a posteriori error estimate

$$
\begin{aligned}
& h_{m(i)}^{1 / 2} \min \left\{1, \frac{1}{\bar{\kappa}_{h, m(i)} h_{m(i)}}\right\}^{1 / 2}\left|\left[u_{h}^{\prime}\right]_{x_{i}}\right|+h_{i} \min \left\{1, \frac{1}{\bar{\kappa}_{h, i} h_{i}}\right\}\left\|r_{h}\right\|_{L^{2}\left(K_{i}\right)} \\
& \quad \leqslant 10 \sum_{j=i}^{i+1}\left\{\left(1+\Gamma\left(\bar{\kappa}_{h, j}, \kappa ; K_{j}\right)\right)\left\|u-u_{h}\right\|_{\kappa, K_{j}}+\left\|\frac{f-f_{h}}{\bar{\kappa}_{h, j}}\right\|_{L^{2}\left(K_{j}\right)}+\left\|\frac{\gamma-\bar{\gamma}_{h, j}}{\bar{\gamma}_{h, j}} \bar{\kappa}_{h, j} u_{h}\right\|_{L^{2}\left(K_{j}\right)}\right\}
\end{aligned}
$$

holds for $i=1, \ldots, N$. Here, $\Gamma\left(\bar{\kappa}_{h, j}, \kappa ; K_{j}\right):=\left\|\frac{\bar{\kappa}_{h, j}-\kappa}{\bar{\kappa}_{h, j}}\right\|_{L^{\infty}\left(K_{j}\right)}$.

Proof. We rewrite the error representation (5.1) in the form

$$
\sum_{i=1}^{N}\left[u_{h}^{\prime}\right]_{x_{i}}\left(v-v_{h}\right)\left(x_{i}\right)-\int_{\Omega} r_{h}\left(v-v_{h}\right)=B\left(u-u_{h}, v\right)-\int_{\Omega}\left\{\left(f-f_{h}\right) v-\left(\gamma-\gamma_{h}\right) u_{h} v\right\} .
$$

Choose $v=\chi_{i} r_{h}$ and $v_{h}=0$, where $\chi_{i}(x)=\left(x_{i}-x\right)\left(x-x_{i-1}\right)$ for $x \in K_{i}$ and $\chi_{i}(x)=0$ otherwise. By explicit calculation we obtain

$$
\left\|\chi_{i}\right\|_{L^{1}\left(K_{i}\right)}=\frac{1}{6} h_{i}^{3}, \quad\left\|\chi_{i}\right\|_{L^{2}\left(K_{i}\right)}=\frac{1}{\sqrt{30}} h_{i}^{5 / 2}, \quad\left\|\chi_{i}^{\prime}\right\|_{L^{2}\left(K_{i}\right)}=\frac{1}{\sqrt{3}} h_{i}^{3 / 2} .
$$

If $\gamma_{h}=\bar{\gamma}_{h}$, then $r_{h}$ is constant $\left(r_{h}=-f_{h}\right.$ or $\left.r_{h}=b_{h}-f_{h}\right)$ and therefore

$$
\begin{aligned}
h_{i}\left\|r_{h}\right\|_{L^{2}\left(K_{i}\right)}^{2}= & \frac{6}{h_{i}} \int_{K_{i}}\left|r_{h}\right|^{2} \chi_{i}=\frac{6}{h_{i}} \int_{K_{i}} r_{h} v \\
\leqslant & \frac{6}{h_{i}}\left(\left\|\left(u-u_{h}\right)^{\prime}\right\|_{L^{2}\left(K_{i}\right)} \frac{h_{i}}{\sqrt{3}}+\left\|\kappa\left(u-u_{h}\right)\right\|_{L^{2}\left(K_{i}\right)}\left(1+\Gamma\left(\bar{\kappa}_{h, j}, \kappa ; K_{j}\right)\right) \frac{\bar{\kappa}_{h, i} h_{i}^{2}}{\sqrt{30}}\right. \\
& \left.+\left(\left\|\frac{1}{\bar{\kappa}_{h, i}}\left(f-f_{h}\right)\right\|_{L^{2}\left(K_{i}\right)}+\left\|\frac{\gamma-\gamma_{h}}{\bar{\gamma}_{h, i}} \bar{\kappa}_{h, i} u_{h}\right\|_{L^{2}\left(K_{i}\right)}\right) \frac{\bar{\kappa}_{h, i} h_{i}^{2}}{\sqrt{30}}\right)\left\|r_{h}\right\|_{L^{2}\left(K_{i}\right)}
\end{aligned}
$$


if $\bar{\kappa}_{h, i} h_{i} \geqslant 1$. This gives

$$
\begin{aligned}
h_{i}\left\|r_{h}\right\|_{L^{2}\left(K_{i}\right)} \leqslant 4 & \left(\left(1+\Gamma\left(\bar{\kappa}_{h, i}, \kappa ; K_{i}\right)\right)\left\|u-u_{h}\right\|_{\kappa ; K_{i}}+\left\|\frac{f-f_{h}}{\bar{\kappa}_{h, i}}\right\|_{L^{2}\left(K_{i}\right)}+\left\|\frac{\gamma-\gamma_{h}}{\bar{\gamma}_{h, i}} \bar{\kappa}_{h, i} u_{h}\right\|_{L^{2}\left(K_{i}\right)}\right) \\
& \cdot \max \left\{1, \bar{\kappa}_{h, i} h_{i}\right\}
\end{aligned}
$$

and the estimate for the $r_{h}$-part is obtained by dividing through $\max \left\{1, \bar{\kappa}_{h, i} h_{i}\right\}=1 / \min \{1$, $\left.1 /\left(\bar{\kappa}_{h, i} h_{i}\right)\right\}$.

Now fix $i \in\{1, \ldots, N\}$ and take $v=\left[u_{h}^{\prime}\right]_{x_{i}} \phi_{i}$ and $v_{h}=0$, where $\phi_{i}$ is the continuous piecewise linear function with $\phi_{i}\left(x_{i}\right)=1$ and $\phi_{i}(x)>0$ if and only if $x \in\left(x_{i}-\delta_{i} h_{i}, x_{i}+\right.$ $\left.\delta_{i+1} h_{i+1}\right)$ where $\delta_{j}:=\min \left\{1,1 /\left(\bar{\kappa}_{h, j} h_{j}\right)\right\}$. We find, if $\bar{\kappa}_{h, j} h_{j} \geqslant 1$, that $\delta_{j} h_{j}=1 / \bar{\kappa}_{h, j}$ and thus

$$
\begin{aligned}
{\left[u_{h}^{\prime}\right]_{x_{i}}^{2} \leqslant \sum_{j=i}^{i+1} } & \left(\left\|\left(u-u_{h}\right)^{\prime}\right\|_{L^{2}\left(K_{j}\right)}+\left(1+\Gamma\left(\bar{\kappa}_{h, j}, \kappa ; K_{j}\right)\right)\left\|\kappa\left(u-u_{h}\right)\right\|_{L^{2}\left(K_{j}\right)}\right. \\
& \left.+\left\|\frac{f-f_{h}}{\bar{\kappa}_{h, j}}\right\|_{L^{2}\left(K_{j}\right)}+\left\|\frac{\gamma-\gamma_{h}}{\bar{\gamma}_{h, j}} \bar{\kappa}_{h, j} u_{h}\right\|_{L^{2}\left(K_{j}\right)}+\frac{1}{\bar{\kappa}_{h, j}}\left\|r_{h}\right\|_{L^{2}\left(K_{j}\right)}\right) \bar{\kappa}_{h, j}^{1 / 2}\left|\left[u_{h}^{\prime}\right]_{x_{i}}\right| .
\end{aligned}
$$

We now use $\bar{\kappa}_{h, j} \leqslant \bar{\kappa}_{h, m(i)}=\left(1 / h_{m(i)}\right) \bar{\kappa}_{h, m(i)} h_{m(i)}$, the previous estimate for $\left\|r_{h}\right\|_{L^{2}\left(K_{j}\right)}$ and this establishes the required bound. Note that the technique to use $\phi_{i}$ has been used in [21] and $[2]$.

Remark 5.1. Theorem 5.2 gives a lower bound for the error in terms of the residual $r_{h}$ in case $\gamma_{h}=\bar{\gamma}_{h}$. This can be generalised to the case $\gamma_{h} \neq \bar{\gamma}_{h}$ (with the same technique) if we split the residual $r_{h}=b_{h}-f_{h}-\left(\gamma_{h}-\bar{\gamma}_{h}\right) u_{h}$ and if we treat $\left(\gamma_{h}-\bar{\gamma}_{h}\right) u_{h}$ as a data error term. However, this estimate is trivial on $V_{h}^{2}$, since the data error terms, especially the one for $\gamma$, dominate the residual terms.

\subsection{Grid modification}

In view of the previous results, we have to guarantee that we can construct a sequence of grids such that $C_{\mathrm{I}}$ satisfies a uniform bound. For practical purposes, we formulate this as a modification of an arbitrary grid.

Theorem 5.3. Let $\mathcal{K}$ be a decomposition of $[0,1]$ and let $\bar{\kappa}_{h}$ be piecewise constant function on $\mathcal{K}$. Then we can modify $\mathcal{K}$ to a new grid $\mathcal{K}^{\text {mod }}$ with the same number of intervals and the same set of constant values for $\bar{\kappa}_{h}^{\text {mod }}$ and such that $C_{\mathrm{I}} \leqslant 2$.

Proof. We start checking $\left|\sin \left(\bar{\kappa}_{h, i} h_{i}\right)\right| \geqslant 1 / 2$ where $i \in \bar{\Lambda}_{-}, \bar{\kappa}_{h, i} h_{i} \geqslant \pi / 2$ for $i=1,2, \ldots, N+1$ (see (3.7)). If $\left|\sin \left(\bar{\kappa}_{h, i} h_{i}\right)\right|<1 / 2$ is detected, we consider $J_{i}:=\left[x_{i-1}, x_{i+1}\right]$ for $i<n$ and $J_{n}:=\left[x_{n-2}, x_{n}\right]$ for $i=n$, and our aim is to bisect $J_{i}$ at an intermediate point (close to $x_{i}$ ) such that both new intervals have the required property. To formalise this problem it is notationally convenient to assume that $J=[0,1]$, to split $J$ at a barycentric coordinate $\xi$, and use indices ' 1 ' and ' 2 ' to refer to quantities on the intervals $[0, \xi]$ and $[\xi, 1]$, respectively. In this setting we have to find $\xi \in[0,1]$ such that $g(\xi):=\min \left\{g_{1}(\xi), g_{2}(\xi)\right\} \geqslant 1 / 2$, where $g_{1}(\xi):=\left|\sin \left(\max \left\{\kappa_{1} \xi, \pi / 2\right\}\right)\right|$ and $g_{2}(\xi):=\left|\sin \left(\max \left\{\kappa_{2} \xi, \pi / 2\right\}\right)\right|$ with $\kappa_{1}:=\bar{\kappa}_{h, i}\left(h_{i}+h_{i+1}\right)$ and $\kappa_{2}:=\bar{\kappa}_{h, i+1}\left(h_{i}+h_{i+1}\right)$. The barycentric location of $x_{i}$ in $J_{i}$ is denoted by $\xi_{0}:=h_{i} /\left(h_{i}+\right.$ $h_{i+1}$ ). Let us assume that $i+1 \in \Lambda^{-}$(otherwise $g_{2}$ is not needed) and that $\kappa_{1} \geqslant \kappa_{2}$ (otherwise we start the following argument with $\kappa_{2}$ ). 

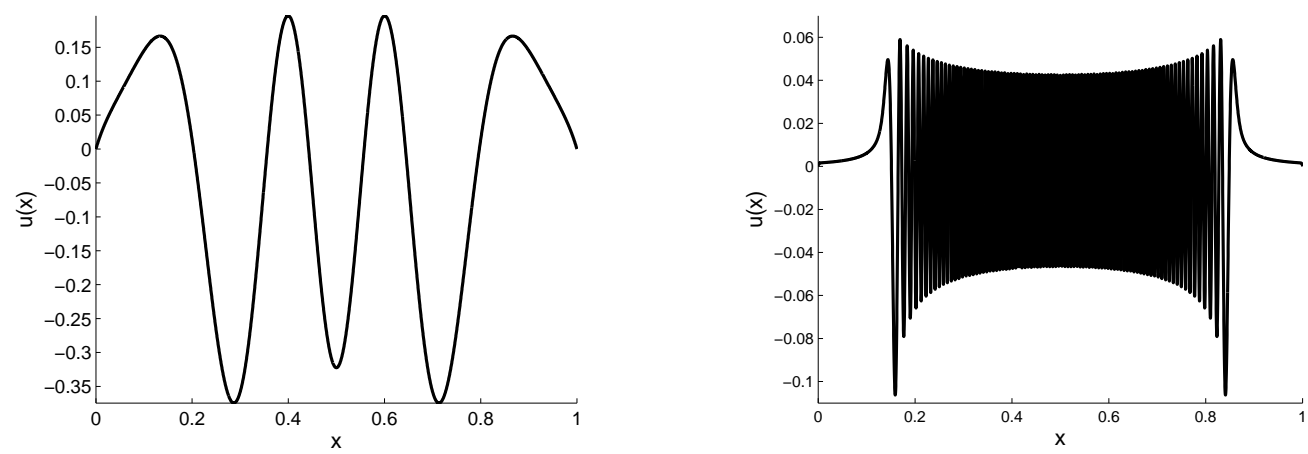

Figure 6.1. Reference solutions for the cases $\left[c_{a}, f_{a}\right]=[1.0 \mathrm{e} 3,6.4 \mathrm{e} 1]$ (left) and $\left[c_{a}, f_{a}\right]=[1.0 \mathrm{e} 6,2.0 \mathrm{e} 3]$ (right)

We introduce the sets $I_{k}^{<}:=\left\{\eta \in J: g_{k}(\eta)<1 / 2\right\}$ and $I_{k}^{>}:=\left\{\eta \in J: g_{k}(\eta) \geqslant 1 / 2\right\}$. By assumption we have $g_{1}\left(\xi_{0}\right)<1 / 2$ and $\kappa_{1} \xi_{0}>\pi / 2$, hence $\xi_{0} \in I_{k}^{<}$. If $\kappa_{2} \leqslant 5 /(6 \pi)$, then $g_{2}(\xi) \geqslant 1 / 2$ for all $\xi \in J$, hence $I_{2}^{>}=J$. Otherwise there exists at least one connected component of $I_{2}^{>}$of length $\pi /\left(2 \kappa_{2}\right)$ in $J$. We consider the component that is closest to $\xi_{0}$. Since it is of length $\pi /\left(2 \kappa_{2}\right) \geqslant \pi /\left(2 \kappa_{1}\right)$, it must contain a connected component of $I_{1}^{>}$and there we find a required $\xi$, for example the one with minimal distance to $\xi_{0}$.

\section{Numerical results}

We consider a sequence of examples with

$$
\gamma(x)=c_{a}\left(2(2 x-1)^{2}-1\right) e^{(x-1 / 2)^{2}}, \quad f(x)=f_{a},
$$

where we take values

$$
\left[c_{a}, f_{a}\right] \in\{[1.0 \mathrm{e} 3,6.4 \mathrm{e} 1],[1.0 \mathrm{e} 4,2.0 \mathrm{e} 2],[1.0 \mathrm{e} 5,6.4 \mathrm{e} 2],[1.0 \mathrm{e} 6,2.0 \mathrm{e} 3]\} .
$$

The potentials are negative on $\Omega_{-}:=(1 / 2-1 / 2 \sqrt{2}, 1 / 2+1 / 2 \sqrt{2})$ and nonnegative on $\Omega \backslash \Omega_{-}$, therefore the solutions of the problems are oscillating with variable frequency in $\Omega_{-}$and exponentially decreasing in $\Omega \backslash \Omega_{-}$. Note that $f_{a} / \sqrt{c_{a}}$ is of similar size in these examples.

Since we have no exact solution, we provide as a reference the numerical solution on a uniform grid with 4164 intervals in $V_{h}^{2}$ (default) or $\mathbb{P}_{2}(\mathcal{K})$ (if indicated). The errors are integrated on the intervals of the fine grid by a trapezoidal rule with 50 points. The reference solutions for $\left[c_{a}, f_{a}\right]=[1.0 \mathrm{e} 3,6.4 \mathrm{e} 1]$ and $\left[c_{a}, f_{a}\right]=[1.0 \mathrm{e} 6,2.0 \mathrm{e} 3]$, obtained as described above, are shown in Fig. 6.1.

As seen from the a priori estimate (Theorem 3.1) and the a posteriori estimate (Theorem 5.1) one might have problems if $\bar{\kappa}_{i} h_{i}$ is close to $\pi \mathbb{N} \backslash\{0\}$ for at least one index $i$. In Section 5.4 we proposed a method to guarantee $\left|\sin \left(\bar{\kappa}_{i} h_{i}\right)\right| \geqslant 1 / 2$. We show calculations that compare the unmodified with the modified grid on a sequence of uniformly refined grids for the example $\left[c_{a}, f_{a}\right]=[1.0 \mathrm{e} 6,2.0 \mathrm{e} 3]$ in Fig. 6.2. In the following we will always use this grid correction.

Let us now compare the solutions in $V_{h}^{1}$ and $V_{h}^{2}$ to solutions from standard finite element spaces $\mathbb{P}_{1}(\mathcal{K})$ and $\mathbb{P}_{2}(\mathcal{K})$. In Figs. 6.3 and 6.4 we see on the left that both methods give comparable results for $\left[c_{a}, f_{a}\right]=[1.0 \mathrm{e} 3,6.4 \mathrm{e} 1]$, while we observe on the right that for $\left[c_{a}, f_{a}\right]=$ $[1.0 \mathrm{e} 6,2.0 \mathrm{e} 3]$ only the approach in $V_{h}^{k}$ gives acceptable results (solid and dashed lines). 

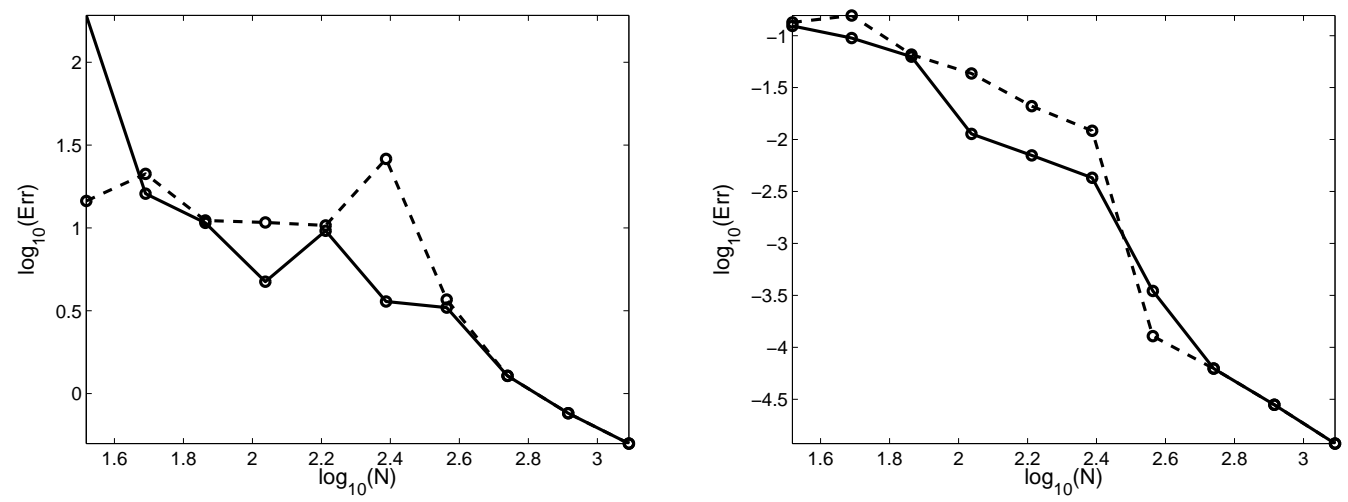

Figure 6.2. Errors on a sequence of solutions in $V_{h}^{p}$ on uniform grids (dashed) vs. those on a corrected grid (solid) for $\left[c_{a}, f_{a}\right]=[1.0 \mathrm{e} 6,2.0 \mathrm{e} 3]$ and $p=1$ (left), $p=2$ (right)
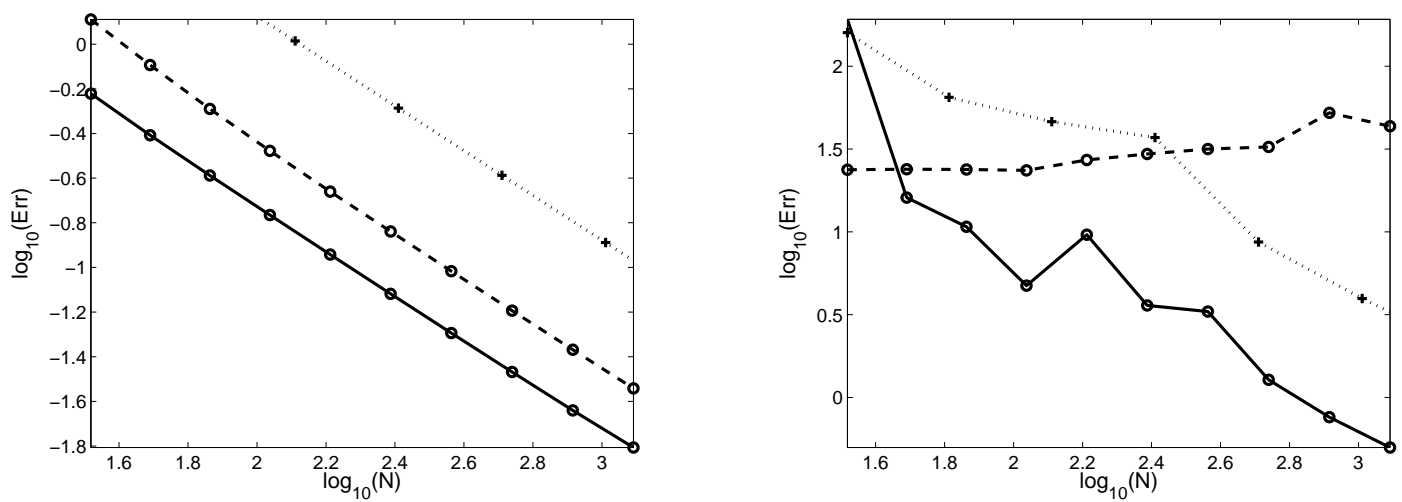

Figure 6.3. Compare errors in $V_{h}^{1}$ (solid) to errors in $\mathbb{P}_{1}(\mathcal{K})$ (dashed) for $\left[c_{a}, f_{a}\right]=[1.0 \mathrm{e} 3,6.4 \mathrm{e} 1]$ (left) and $\left[c_{a}, f_{a}\right]=[1.0 \mathrm{e} 6,2.0 \mathrm{e} 3]$ (right) on a sequence of uniform grids. The dotted line shows the a posteriori error on $V_{h}^{1}$
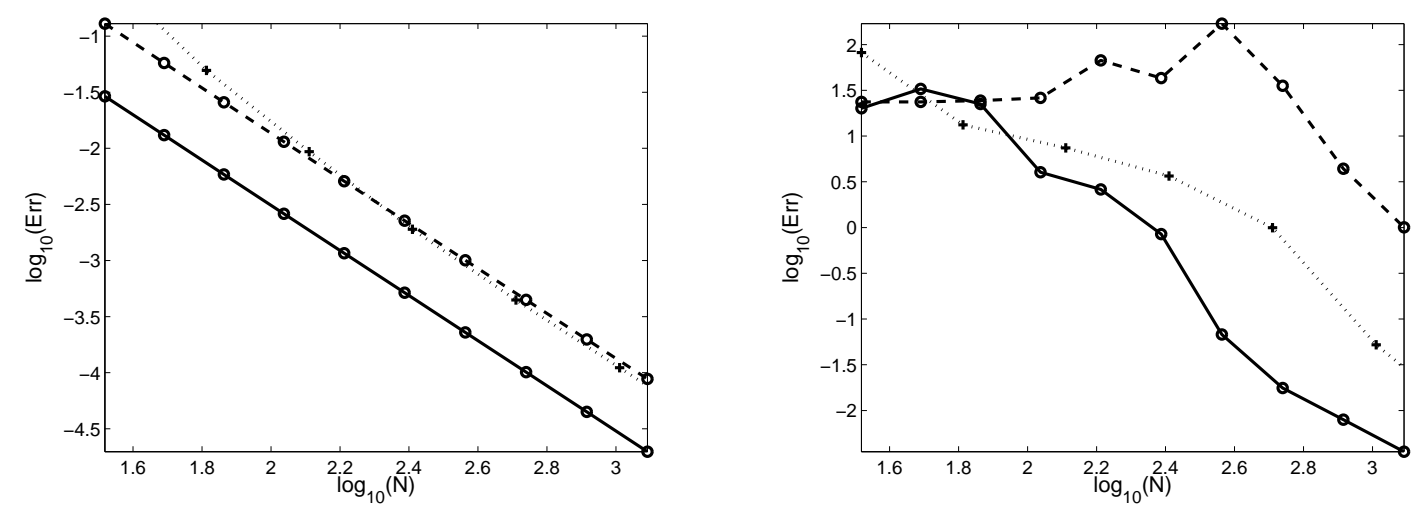

Figure 6.4. Compare errors in $V_{h}^{2}$ (solid) to errors in $\mathbb{P}_{2}(\mathcal{K})$ (dashed) for $\left[c_{a}, f_{a}\right]=[1.0 \mathrm{e} 3,6.4 \mathrm{e} 1]$ (left) and $\left[c_{a}, f_{a}\right]=[1.0 \mathrm{e} 6,2.0 \mathrm{e} 3]$ (right) on a sequence of uniform grids. The dotted line shows the a posteriori error on $V_{h}^{2}$ 

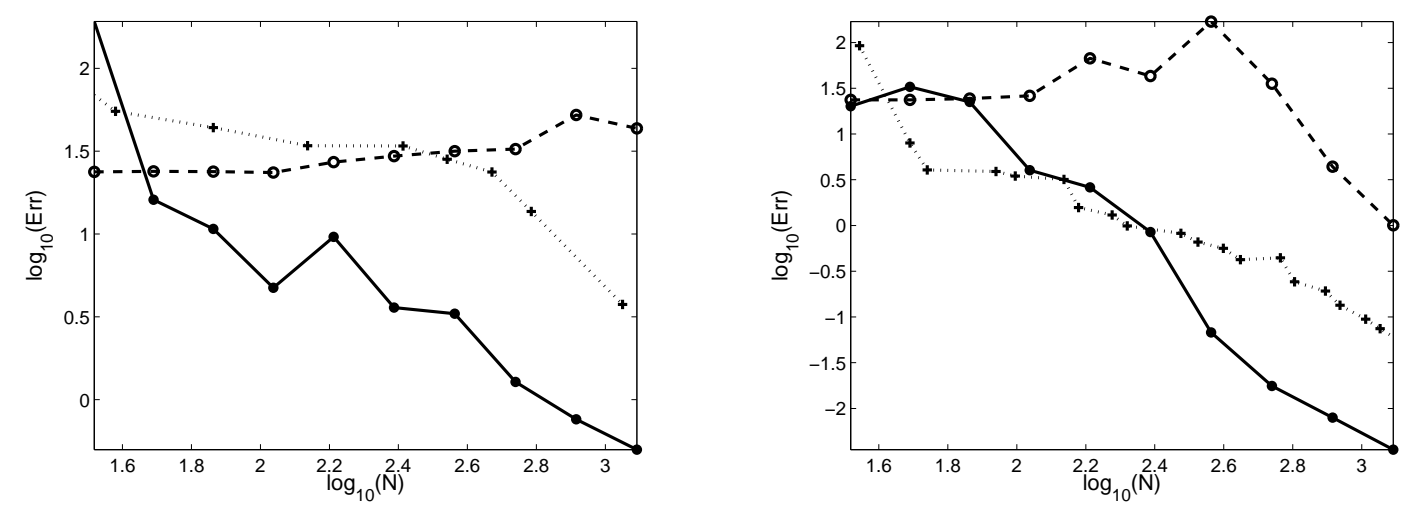

Figure 6.5. The adaptive finite element method in $V_{h}^{1}$ (left) and $V_{h}^{2}$ (right) for $\left[c_{a}, f_{a}\right]=[1.0 \mathrm{e} 6,2.0 \mathrm{e} 3]$ and its error estimates (dotted). The others curves are those from Fig. 6.3 and 6.4.

Furthermore, we show the results of the a posteriori error estimates in Theorem 5.1 on the sequence of uniform grids in Figs. 6.3 and 6.4. Although there is an overestimation by a factor of about 10, the dependence on the number of unknowns is correct and this factor holds for both small and large $c_{a}, f_{a}$.

Finally, we want to use our a posteriori error estimates to establish an adaptive finite element algorithm. One starts with some coarse grid and then performs a sequence of steps solve-estimate-refine [4] until the error estimate is below a prescribed tolerance. Here we assume that 'solve' returns the exact discrete solution and that the the step 'refine' returns the exact evaluations of the right-hand side in the a posteriori error estimates. The procedure 'refine' first performs a marking of the elements as in [4] (a fixed energy fraction strategy with $\theta=0.7$ ) and by bisecting all marked elements into two equal parts. Recall that we include after this refinement step our grid correction step from Section 5.4. We stop the procedure when the estimated error is below 0.01 .

The results in Fig. 6.5 show that the adaptive algorithms follow a curve in the $N \mapsto$ $\log _{10}(\operatorname{err}(N))$-diagram that is in accordance to the previous observations on uniform grids. The algorithm behaves as expected, taking the overestimation of the error into account. Observe that our example is especially bad for adaptivity since the right-hand side is constant, so that the residuals do not vary strongly. Furthermore we see that the number of adaptive steps is quite large, or, the gain per step is quite low. This could be a consequence of the large constants that appear in the estimates between the exact and the estimated error due to the theoretical results in $[18$, Thm. 5.2].

One should note the difference between the polynomial and fitted elements. In norms, Verfürth's [21] and our estimate behave the same, however, in the computations, the adaptive algorithm will not work properly on the polynomial spaces (Fig. 6.6) since in this case the residual will be small only if $h$ is sufficiently small. This is illustrated by the fact that the adaptive error curves follow the a priori error curves that do not show convergence in the considered range of $h$. 

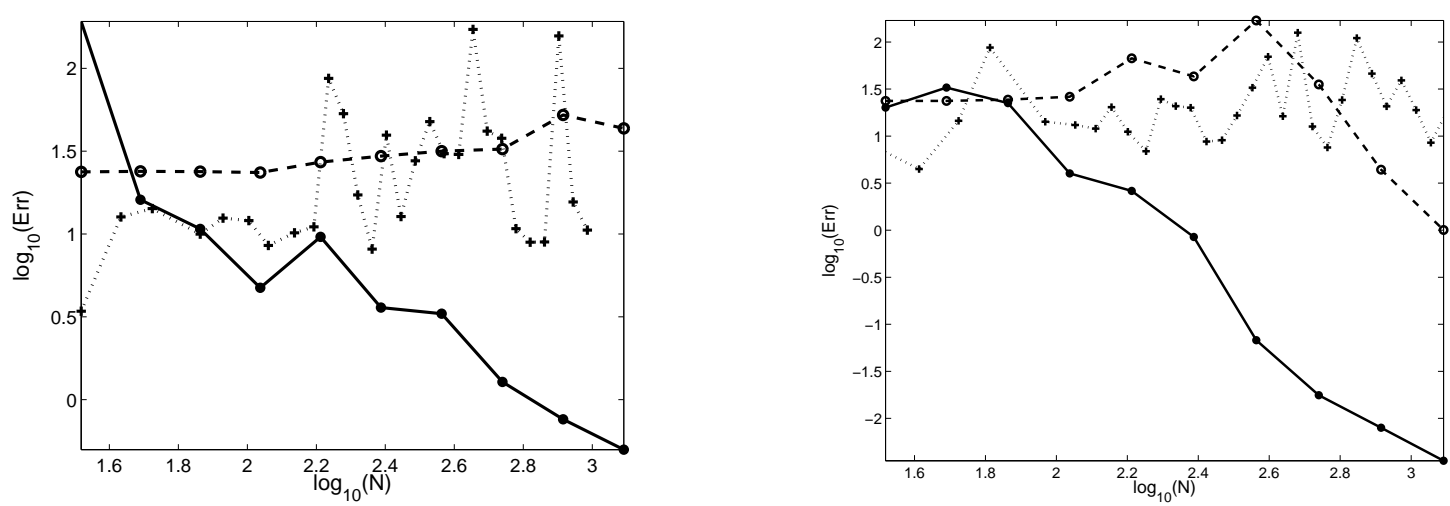

Figure 6.6. The adaptive finite element method in $\mathbb{P}_{1}(\mathcal{K})$ (left) and $\mathbb{P}_{2}(\mathcal{K})$ (right) for $\left[c_{a}, f_{a}\right]=[1.0 \mathrm{e} 6,2.0 \mathrm{e} 3]$ and its error estimates (dotted). The others curves are those from Fig. 6.3 and 6.4.

\section{Conclusion}

For boundary value problems like (2.1) with large (negative) $\gamma$ the finite element approach with polynomial ansatz functions leads to reasonable approximations only if $h \sqrt{|\gamma|}<1$ which is a strong condition in applications. This deficiency is also visible in the adaptive finite element approach. We proposed a finite element method where the basis functions have been replaced by local solutions of the constant coefficient problem. These functions are well defined if the grids are corrected suitably. Using adapted interpolation estimates we derive a priori and a posteriori error estimates that do not have such a strong demand on the grid refinement. The results show that these new basis functions are beneficial for uniform as well as adaptive meshes.

This method can be implemented in existing finite element codes, if one replaces the subroutines that evaluate basis functions and the routines that do quadrature accordingly. However, these routines have to be implemented very carefully considering the various parameter ranges in their arguments. While provided explicit formulas here, one might in general use quadrature methods for oscillating integrals [12] [9].

Here, we restricted ourselves to the first and second order approach. A generalisation to higher order is possible, for example with the iterative technique developed in [19].

\section{References}

[1] S. Adjerid, M. Aiffa, and J. E. Flaherty, High-order finite element methods for singularly perturbed elliptic and parabolic problems, SIAM J. Appl. Math., 55 (1995), pp. 520-543.

[2] M. Ainsworth and I. Babuška, Reliable and robust a posteriori error estimation for singularly perturbed reaction diffusion problems, SIAM J. Numer. Anal., 36 (1999), pp. 331-353.

[3] I. Babuška and J. M. Melenk, The partition of unity method, Int. J. Numer. Meth. Engng., 40 (1996), pp. $727-758$.

[4] W. Dörfler, A convergent adaptive algorithm for Poisson's equation, SIAM J. Numer. Anal., 33 (1996), pp. 1106-1124.

[5] W. Dörfler, Uniform error estimates for an exponentially fitted finite element method for singularly perturbed elliptic equations, SIAM J. Numer. Anal., 36 (1999), pp. 1709-1739.

[6] A. Ern and J.-L. Guermond, Theory and practice of finite elements, vol. 159 of Applied Mathematical Sciences, Springer, New York, 2004. 
[7] P. W. Hemker, A Numerical Study of Stiff Two-Point Boundary Problems, Ph.D. thesis, Mathematisch Centrum, Amsterdam, 1977.

[8] D. Hollevoet, M. Daele, and G. Berghe, The optimal exponentially-fitted Numerov method for solving two-point boundary value problems, J. Comput. Appl. Math., 230 (2009), pp. 260-269.

[9] D. Huybrechs and S. Olver, Highly oscillatory quadrature, in: Highly oscillatory problems (B. Enquist et al., ed.), Cambridge University Press, Cambridge, 2009, pp. 25-50.

[10] F. Ihlenburg, Finite Element Analysis of Acoustic Scattering, Springer, Berlin, 1998.

[11] F. Ihlenburg and I. Babushka, Finite element solution of the Helmholtz equation with high wave number. Part I: The h-version of the FEM, Computers Math. Applic., 30 (1995), pp. 9-37.

[12] L. G. Ixaru, Operations on oscillatory functions, Computer Physics Communications, 105 (1997), pp. 1-19.

[13] J. M. Melenk and S. Sauter, Wave-number explicit convergence analysis for Galerkin discretizations of the Helmholtz equation, 2009, ASC Report 31/2009.

[14] M. Melenk, hp-finite element methods for singular perturbations, Springer, Berlin, 2002.

[15] A. Moiola, R. Hiptmair, and I. Perugia, Plane wave approximation of homogeneous Helmholtz solutions, 2010, i.M.A.T.I.-C.N.R., 25PV10/23/0, 1-35.

[16] A. H. Schatz, An observation concerning Ritz-Galerkin methods with indefinite bilinear forms, Math. Comput., 28 (1974), pp. 959-962.

[17] C. Schwab, p- and hp-finite element methods. Theory and applications in solid and fluid mechanics, Clarendon Press, Oxford, 1998.

[18] R. Stevenson, Optimality of a standard adaptive finite element method, Found. Comput. Math., 7 (2007), pp. 245-269.

[19] W. G. Szymczak and I. Babuška, Adaptivity and error estimation for the finite element method applied to convection diffusion problem, SIAM J. Numer. Anal., 21 (1984), pp. 910-954.

[20] R. Tezaur and C. Farhat, Three-dimensional discontinuous Galerkin elements with plane waves and Lagrange multipliers for the solution of mid-frequency Helmholtz-problems, Int. J. Numer. Meth. Engng., 66 (2006), pp. 796-815.

[21] R. Verfürth, Robust a posteriori error estimators for the singularly perturbed reaction-diffusion equation, Numer. Math., 78 (1998), pp. 479-493. 